\title{
Acteoside Repressed Microglia M1 Polarization Through Inhibited NF-kB Signalling Pathway and AMPK-Mediated Mitochondria Function Recovery
}

Ying-Qi Li

China Pharmaceutical University

Yi Chen

China Pharmaceutical University

Si-Qi Jiang

China Pharmaceutical University

Yuan-Yuan Shi

China Pharmaceutical University

Xiao-Li Jiang

China Pharmaceutical University

Shan-Shan Wu

China Pharmaceutical University

Ping Zhou

China Pharmaceutical University

Hui-Ying Wang

China Pharmaceutical University

Ping Li

China Pharmaceutical University

Fei Li ( $\square$ lifeicpu@163.com)

China Pharmaceutical University https://orcid.org/0000-0001-8721-5183

Research

Keywords: acteoside, BV-2 cells, metabolism, RNA-seq, mitochondria, neuroinflammation.

Posted Date: December 16th, 2020

DOI: https://doi.org/10.21203/rs.3.rs-127246/v1

License: (c) (1) This work is licensed under a Creative Commons Attribution 4.0 International License.

Read Full License 


\section{Abstract}

Background: Alzheimer's disease (AD) is the most frequent type of dementia. While acteoside (ACT), a compound isolated from Cistanche tubulosa, possesses neuroprotective properties. However, the underlying mechanism in regulating microglia polarization remains ill-defined.

Methods: Herein, $\mathrm{AlCl}_{3}$-induced $\mathrm{AD}$ model in zebrafish larvae was applied to uncover the therapeutic efficacy of ACT. BV-2 cells were used to demonstrate the role of ACT on microglia polarization. RNASequence, HPLC-Q-TOF-MS, western blot and molecular docking were combined to confirm its mechanism.

Results: ACT significantly ameliorated the experimental dyskinesia and nervous system disorders in zebrafish. Subsequently, it suppressed M1 polarization and promoted to the M2 phenotype in LPSinduced BV-2 cells. We first demonstrated that ACT exerted profound transcriptomic impact, which involved regulation of key signaling pathways in inflammation, arginine biosynthesis, as well as pantothenate and CoA biosynthesis, correlating with mitochondria function. ACT treatment reduced microglia M1 polarization by inhibiting the NF-KB signalling pathway. And the metabolic pathways were further confirmed by HPLC-Q-TOF-MS. In addition, ACT rectified excessive ROS to restore mitochondria function through AMPK-mediated PGC-1a and UCP-2 upregulation, consistent with metabolic changes. Intriguingly, ACT may directly bind to both NF-KB and AMPKa, as evidenced by molecular docking.

Conclusions: The research provided an infusive mechanism of ACT and illustrated a new perspective based on mitochondrial dysfunction to reveal the connection between metabolism and microglia polarization.

\section{Background}

Alzheimer's disease $(A D)$ is a common neurodegenerative disease accompanied with cognitive impairment and dyskinesia ${ }^{[1]}$. It's characterized by severe neuronal loss, senile plaques, and neurofibrillary tangles ${ }^{[2]}$. The pathogenesis of $A D$ is multidimensional and links to neuroinflammation.

Neuroinflammation is driven by the activation of glial cells, closely related to the development of $A D^{[3,4]}$. During the progression and exacerbation of neuroinflammation, microglia is considered as the key factor.

Microglia are the primary immune cells in the central nervous system. It's closely associated with a cascade of processes, containing brain development, maintaining neural environment, as well as responses to injury and repair ${ }^{[1]}$. Moreover, microglia can be stimulated to an M1 phenotype and the expression of pro-inflammatory cytokines are increased when neuroinflammation-related diseases such as $A D$ occurred ${ }^{[5]}$. Studies also demonstrate that the polarization to M1 phenotype is often accompanied with metabolic disorders ${ }^{[6]}$, causing energy metabolism imbalance and mitochondrial dysfunction ${ }^{[7]}$. These adverse changes are derived to neurodegeneration, even AD. 
Acteoside (ACT), a phenylethanoid glycoside, is primarily derived from Cistanche tubulosa. Increasing evidence has suggested that ACT possessed numerous pharmacological activities, including neuroprotective ${ }^{[8]}$, anti-inflammatory ${ }^{[9]}$ and antioxidant ${ }^{[10]}$ effects. Particularly, ACT has been reported to improve learning and memory impairment as well as upregulate energy metabolism in streptozotocininduced rats ${ }^{[11]}$. It also has been suggested to inhibit neuronal apoptotic cell death and mitochondrial damage in the experimental autoimmune encephalomyelitis mice ${ }^{[12]}$. However, fewer studies have been focused on the effect of ACT on microglia M1/M2 polarization. Especially, various mechanisms, such as repair of mitochondria function and the regulation of cell metabolism, have not been performed. In addition, the mechanism of ACT contributed to microglia M1/M2 polarization has remained unexplored.

The present report was aimed to investigate the therapeutic efficacy of ACT as well as underly molecular mechanism of ACT in AD. Herein, ACT showed significant neuroprotection effect in $\mathrm{AlCl}_{3}$-induced $A D$ zebrafish larvae. In addition, ACT effectively inhibited M1 polarization and promoted the M2 phenotype in LPS-induced BV-2 cells. RNA-Sequencing (RNA-Seq) integrated with metabolomics method to better understand the underlying mechanism of ACT in regulating microglia polarization. The crosstalk between metabolism and microglia polarization in terms of mitochondrial function were investigated. This study will provide a new respect for the further investigation of ACT as a potential therapeutic agent for treating AD.

\section{Methods}

\section{Animals and model grouping}

Wild-type zebrafish (AB strain, 4 months old) were chosen in this study (Nanjing Qi Wu Biotechnology Co., Ltd.). They were maintained under $14 / 10 \mathrm{~h}$ light/dark cycle at $28^{\circ} \mathrm{C}$, following the previous method ${ }^{[13]}$. Natural fertilized and normal developed embryos were generated and cultured to 3 days post-fertilization (dpf) in an illumination incubator. All zebrafish experiments were carried out under the supervision of the Animal Ethics Committee of China Pharmaceutical University.

Zebrafish larvae were divided into six groups and treated from $3 \mathrm{dpf}$ to $7 \mathrm{dpf}$ : control group, model group, model + donepezil hydrochloride (DPZ) group, model + ACT groups. The control group was maintained in the medium with $0.2 \% \mathrm{DMSO}$ and the model group was treated with $150 \mu \mathrm{M} \mathrm{AlCl} 3(\mathrm{pH}$ 5.8). The model + DPZ group was co-treated with $\mathrm{AlCl}_{3}$ and $8 \mu \mathrm{M} \mathrm{DPZ}$. The model + ACT groups were co-treated with $\mathrm{AlCl}_{3}$ and different concentrations of ACT $(200,100,50 \mu \mathrm{M})$. ACT (HPLC purity $\geq 98 \%)$ was obtained from Baoji Herbest Bio-Tech Co., Ltd. (Baoji, China). $\mathrm{AlCl}_{3} \cdot 6 \mathrm{H}_{2} \mathrm{O}$ and DPZ were purchased from Shanghai Aladdin Bio-Chem Technology Co., LTD. (Shanghai, China).

\section{Behavioral analysis}


Zebrafish larvae movements were recorded with a ViewPoint behavioral analyzer (Zebralab 2018, ViewPoint Life Sciences Co., Ltd.) at $28^{\circ} \mathrm{C}$. Briefly, the behavioral parameters and result processing were consistent with the method we established earlier ${ }^{[13]}$. Here, average speed (AS), speed change $(\Delta S)$, dyskinesia recovery rate (DRR), and response efficiency (RE, \%) were selected to evaluate dyskinesia recovery in zebrafish.

\section{Determination of acetylcholinesterase (AChE) and choline acetyltransferase (ChAT) activity}

After treated from $3 \mathrm{dpf}$ to $7 \mathrm{dpf}$, zebrafish larvae were collected to measure AChE and ChAT activity. Based on the manufacturer's protocol, the activity was detected by the enzyme-linked immunosorbent assay (ELISA) kits (MLBIO biotechnology Co. Ltd., Shanghai, China). And the protein concentrations of different samples were determined by BCA method.

\section{Cell cultures and treatments}

BV-2 cell line (immortalized murine microglial cell line) was purchased from the American Type Culture Collection (ATCC, Manassas, VA, USA). They were cultured in DMEM (KeyGen Biotech Co., Ltd, Nanjing, China). The medium was supplemented with 10\% FBS (Gibco, Grand Island, NY, USA), $100 \mathrm{U} / \mathrm{mL}$ penicillin, as well as $100 \mathrm{mg} / \mathrm{mL}$ streptomycin, with $95 \%$ air $/ 5 \% \mathrm{CO}_{2}$ at $37^{\circ} \mathrm{C}$.

BV-2 cells were incubated with ACT $(50,25,12.5 \mu \mathrm{M})$ or stimulating with Lipopolysaccharide (LPS, 1 $\mu \mathrm{g} / \mathrm{mL}$; Sigma-Aldrich, St Louis, MO, USA) for $24 \mathrm{~h}$. Finally, all the cells or supernatant were collected for the various analyses.

\section{Cell viability assay}

Cell Counting Kit-8 (CCK-8) assay (JianCheng Bioengineering Institute, Nanjing, China) was used to evaluate the viability of BV-2 cells. The cells were seeded in a $96-$ well plate $\left(1 \times 10^{4}\right.$ cells/well, Wuxi NEST Biotechnology Co., Ltd.). Briefly, the medium was removed at the end of the treatment, and $100 \mu \mathrm{L}$ of serum-free medium containing CCK-8 solution was added to each well for $2 \mathrm{~h}$ at $37^{\circ} \mathrm{C}$. The absorbance was measured at $450 \mathrm{~nm}$ with a microplate reader (Bio-Tek Instrument, Winooski, VT, USA). Cell viability is expressed as a percentage of the control group. The experiment was repeated three times.

\section{Nitric oxide (NO) production assay}

NO was determined by measuring nitrite levels in the BV-2 culture supernatant using Griess reagent. Briefly, at the end of the treatment, the medium $(100 \mu \mathrm{L})$ was transferred to a new 96 -well plate. The same 
volume of Griess reagent was added to each well and reacted for 15 min in the dark. The absorption at $540 \mathrm{~nm}$ was determined by a Microplate Reader.

\section{Inflammatory cytokines levels in supernatant}

The concentrations of TNF- $\mathrm{a}, \mathrm{IL}-1 \beta$ and IL-10 in BV-2 cell supernatant were determined by ELISA kits according to the manufacturer's instructions (MLBIO biotechnology Co. Ltd., Shanghai, China).

\section{Observation of cellular morphology}

To determine the effect of ACT on BV-2 cells M1/M2 polarization, the cells were plated in 6-well dish and observed under the inverted microscope (Nikon ECLIPSE Ti2, Japan).

\section{Cellular metabolism determination by HPLC-Q-TOF-MS analysis}

BV-2 cells were seeded in 6-well dish separately ( $n=6 /$ group). After treatment, the medium was removed, and the cells were washed three times with cold PBS. Then immediately exposed to liquid nitrogen to suppress cells metabolism. The cells were harvested with cold $80 \%$ methanol ( $1 \mathrm{~mL} /$ well) and the suspension were transferred to a $2 \mathrm{~mL}$ Eppendorf tube. To facilitate protein precipitation, vigorously vortexed for $1 \mathrm{~min}$ and centrifuged at 13,000 rpm for $15 \mathrm{~min}$ at $4^{\circ} \mathrm{C}$. The cell suspension was transferred to a new $2 \mathrm{~mL}$ Eppendorf tube and dried under a stream of nitrogen and stored at $-80^{\circ} \mathrm{C}$ until analysis. The dried residue was reconstituted in $150 \mu \mathrm{L}$ of pre-cooled $25 \%$ acetonitrile. In order to ensure the stability and accuracy of the sequence analysis, equal volumes $(10 \mu \mathrm{L})$ of each cell sample were combined as quality control (QC) samples. During metabolite detection, these samples were injected after every six cell samples to confirm their stability. A $1 \mu \mathrm{L}$ aliquot was injected for HPLC-Q-TOF-MS.

HPLC-Q-TOF-MS analysis was performed on Agilent 1290 HPLC system connected with the Aglient 6530 Quadrupole Time-of-Flight (Q-TOF) mass spectrometer (Agilent Technologies, Santa Clara, CA, USA). The separation was carried out on an ACQUITY UPLC BEH C ${ }_{18}$ column $(2.1 \times 100 \mathrm{~mm}, 1.7 \mu \mathrm{m})$. The mobile phase was composed of $0.1 \%$ formic acid-water $(\mathrm{v} / \mathrm{v} ; \mathrm{A})$ and acetonitrile $(\mathrm{B})$.

The flow rate was set at $0.4 \mathrm{~mL} / \mathrm{min}$ with the following optimal gradient elution condition: 0 to $2 \mathrm{~min}, 5 \%$ B; 2 to $20 \mathrm{~min}, 5 \%$ to $95 \%$ B (positive ion mode); 0 to $2 \mathrm{~min}, 5 \% \mathrm{~B} ; 2$ to $20 \mathrm{~min}, 5 \%$ to $95 \% \mathrm{~B}$ (negative ion mode). The operation parameters of the mass spectrometer were set as follows: gas temperature, $320^{\circ} \mathrm{C}$; drying gas, 10 L/min; nebulizer, 35 psi; VCap, 4000 V; fragmentor, $120 \mathrm{~V}$.

The raw data were operated under MassHunter Workstation Software version B.07.00 (Agilent Technologies, Santa Clara, CA, USA). The raw data were pre-processed by XCMS platform. Principal components analysis (PCA) and partial least-squares discriminant analysis (PLS-DA) of the normalized 
data were conducted with MetaboAnalyst (https://www.metaboanalyst.ca). Combined with literature, the differential metabolites (VIP $>1$, T-test $P<0.05$ ) were identified on HMDB (https://hmdb.ca). Finally, pathway analysis was conducted with MetaboAnalyst.

\section{Measurement of mitochondrial membrane potential (MMP)}

MMP was detected using fluorescent probe JC-1 (Beyotime, China) in accordance with the manufacturer's instructions. Briefly, cells from different groups were rinsed with PBS and incubated with JC-1 staining solution for $20 \mathrm{~min}$ at $37^{\circ} \mathrm{C}$. After staining, cells were washed twice using staining buffer. Then, fluorescent signals were detected by flow cytometry (BD Accuri C6).

\section{Measurement of mitochondrial adenosine 5'-triphosphate (ATP)}

ATP concentration in mitochondria was detected by an ATP Assay Kit (Beyotime, China) in accordance with manufacturer's instructions. Briefly, the culture medium of BV-2 cells from different groups were discarded, and cells were homogenized with lysis buffer on ice. The supernatant obtained after centrifugation $(12,000 \mathrm{~g}, 5 \mathrm{~min})$ was used to determine the ATP concentration. The luminescence (luciferase-catalyzed fluorescein reaction) was detected by EnVision Multimode Microplate Reader (PerkinElmer).

\section{Measurement of intracellular reactive oxygen species (ROS) level}

ROS Assay Kit (Beyotime, China) was used to measure ROS level. The cells from different groups were incubated with DCFH-DA $(10 \mu \mathrm{M})$ for $20 \mathrm{~min}$ at $37^{\circ} \mathrm{C}$. After probe loading, cells were washed three times with DMEM. Then, fluorescent signals were detected by flow cytometry (BD Accuri C6).

\section{Transmission electron microscopy (TEM)}

BV-2 cells were seeded in 6-well dish. The medium was removed and $1 \mathrm{~mL}$ of $2.5 \%$ glutaraldehyde was rapidly added to each well. Then the cells were transferred to a $1.5 \mathrm{~mL}$ Eppendorf tube and centrifuged at $1000 \mathrm{rpm}$ for $3 \mathrm{~min}$. The cells were fixed overnight with new $2.5 \%$ glutaraldehyde at $4^{\circ} \mathrm{C}$. After fixation, dehydration and embedding, the cells were observed with an HT7800 transmission electron microscope (Hitachi, Tokyo, Japan).

\section{RNA-seq and bioinformatic data analysis}


Total RNA from BV-2 cells ( $n=3 /$ group) were extracted using Trizol reagent (Vazyme Biotech, China) according to the reagent manufacturer's instructions. All analytical samples were sent to Majorbio (Shanghai Majorbio Bio-pharm Technology Co., Ltd.) for performing the RNA sequence assay. The data were analyzed on the free online platform of Majorbio Cloud Platform (www.majorbio.com). The parameters for the differential expression analysis were $P$-adjust $<0.05$ and $\mid \log 2 \mathrm{FCl} \geq 1$. The original sequence data have been submitted to the database of the NCBI Sequence Read Archive (SRA).

\section{Quantitative real time polymerase chain reaction (qRT-PCR)}

The total RNA of BV-2 cells in each group was harvested using $500 \mu \mathrm{L}$ RNA-easy ${ }^{\mathrm{TM}}$ Isolation Reagent (Vazyme Biotech, China), and reverse transcription reaction was conducted with FastKing-RT SuperMix (TIANGEN Biotech, China). Reactions were performed according to the manufacturer's protocol. cDNA was subjected to qRT-PCR assays with specific primers and TransStart TOP Green qPCR SuperMix (TransGen Biotech, China). The primers are listed in Table S1 (see Additional file 1) and $\beta$-actin was used as the internal control. The $2^{-\triangle \Delta C T}$ method was used for quantitative analysis.

\section{Western blot analysis}

BV-2 cells were lysed by RIPA lysis buffer (KeyGen Biotech Co., Ltd, Nanjing, China) containing 1\% Protease Inhibitor Cocktail (Thermo Fisher) to obtain total protein. A 10\% SDS-PAGE was performed to separate the proteins, which were transferred to NC membranes. After blocking with $5 \%$ skimmed milk/BSA for $2 \mathrm{~h}$, the membranes were incubated with AMPKa (Proteintech), p-AMPKa (Affinity Biosciences), PGC-1a (Proteintech), NF-KB (Proteintech), p-NF-KB (ABclonal) or GAPDH (ABclonal) antibodies in $5 \%$ TBST at $4{ }^{\circ} \mathrm{C}$ overnight. The membranes were incubated with a secondary horseradish peroxidase-conjugated antibody (ABclonal) for $1 \mathrm{~h}$ at room temperature. The high-sig ECL western blotting substrate (Tanon, China), Gel imaging system (Tanon, China) and ImageJ software were used to visualization and quantitation.

\section{Molecular docking}

Molecular docking analysis were performed using Autodock software (Version 4.2). The affinity between ACT and proteins were observed by AutodockTools software. The three-dimensional (3D) protein structures of AMPKa (PDB ID: 5g5j), and NF-KB (PDB ID: 4q3j) were retrieved from the Protein Data Bank (https://www.rcsb.org).

\section{Statistical analysis}

All data are expressed as the mean \pm standard deviation (SD). The differences between the different groups were analyzed by one-way analysis of variance (ANOVA), followed by Tukey's multiple 


\section{Results}

\section{ACT alleviated dyskinesia and improved cholinergic system function in zebrafish larvae}

$\mathrm{AlCl}_{3}$-induced $\mathrm{AD}$ model in zebrafish larvae was used to demonstrate the effect of ACT on AD. Firstly, the zebrafish larvae movement within light/dark cycles was observed and their swimming trails were recorded (Fig. 1a, 1b). AS and $\Delta S$ of zebrafish movement induced by $\mathrm{AlCl}_{3}$ in the corresponding time after administration were calculated. The results showed that different doses of ACT effectively increased the AS and $\triangle S$ of zebrafish (Fig. 1C). DRR and RE uncovered a more intuitive comparison of ACT and DPZ (Fig. 1d). Accordingly, ACT alleviated dyskinesia, exhibiting similar effects as DPZ.

It's generally agreed that the cholinergic system plays an important role in learning and memory processes. Thus, the activities of AChE and ChAT were used to reveal the effect of $\mathrm{ACT}$. $\mathrm{AlCl}_{3}$ exposure in zebrafish rendered with brain cholinergic alteration (Fig. 1e). It was outstanding that ACT treatment suppressed the activity of AChE. In addition, the activity of ChAT exhibited a decrease after ACT treatment. In brief, ACT showed a profound impact on cholinergic system function in $\mathrm{AlCl}_{3}$-induced $\mathrm{AD}$ zebrafish larvae.

\section{ACT suppressed M1 polarization and promoted M2 polarization in LPS-induced BV-2 cells}

The effects of ACT on microglia polarization were studied in vitro using BV-2 microglial cells. LPS significantly decreased BV-2 cell viability after treated $24 \mathrm{~h}$. Fortunately, ACT increased the cell viability of LPS-induced BV-2 cells (Fig. 2a). In addition, the morphology of BV-2 cells was observed. After $24 \mathrm{~h}$ of LPS stimulation, it showed that BV-2 cells underwent a M1 polarization state. And the morphological changes were prevented by ACT co-treatment (Fig. 2b).

Moreover, the results indicated that unlike BV-2 cells stimulated by LPS, BV-2 cells co-treated with ACT displayed significantly suppressed TNF-a (Fig. 2C), IL-1ß (Fig. 2d) and NO (Fig. 2f) expressions in cell supernatant. These are classical pro-inflammatory cytokines as the indicators of M1 microglia polarization. Similar to the results of ELISA, the results of qPCR discovered that TNF-a, nitric oxide synthase (iNOS), IL-1 $\beta$ and CD86 mRNA expressions were significantly inhibited by ACT treatment compared with the LPS group (Fig. 3a).

Furthermore, we measured M2 microglia polarization levels by ELISA (Fig. 2e) and qPCR (Fig. 3b), the results of which indicated that ACT significantly increased M2 microglia-related marker expression levels (IL-10, CD206, TGF- $\beta$ and Arg-1). Taken together, these results showed that ACT suppressed M1 microglia polarization and promoted the M2 phenotype. 
ACT regulated M1/M2 polarization via the inhibition of NF-KB signaling pathway in LPS-induced BV-2 cells

The transcriptomic analysis was performed by RNA-seq to understand the mechanism of ACT in BV-2 cells from an overall level. PCA illustrated that the control, LPS, and ACT groups could be well distinguished (Fig. 4a). It revealed 899 differentially expressed genes (DEGs) between the control group and LPS group, whereas 49 DEGs between the LPS group and ACT group (Fig. 4b). Consistently, Gene ontology (GO) enrichment analysis (Fig. 4c) and Kyoto Encyclopedia of Genes and Genomes (KEGG) enrichment analysis (Fig. 4d) uncovered that the effect of ACT was involved in the NF-KB signalling pathway. The set of genes associated to NF-KB signalling pathway was further confirmed and their homeostasis were certainly affected by LPS. As expected, ACT significantly affected their expressions (Fig. 4e).

NF-KB signalling pathway is a classical pathway to regulate the progression of inflammation, ultimately resulting in the release of pro-inflammatory factors. To gain mechanistic support, key protein of the NF-KB signalling pathway was evaluated by western blot analysis. LPS stimulation led to the activation of NF$\mathrm{KB}$, associated with promoting M1 polarization. Consistent with RNA-seq analysis, ACT inhibited LPSstimulated NF-KB phosphorylation (Fig. 4f). Therefore, ACT relieved the LPS-induced M1 polarization via the NF-KB signalling pathway in BV-2 cells.

\section{ACT impaired arginine biosynthesis as well as pantothenate and CoA biosynthesis in LPS-induced BV-2 cells}

RNA-seq demonstrated that the pathways affected by ACT also included arginine (Arg) biosynthesis as well as pantothenate and CoA biosynthesis (Fig. 4d). And it has been suggested that LPS stimulation causing BV-2 cells metabolism disorders associated with M1 polarization ${ }^{[14]}$. Thus, untargeted cell metabolome by HPLC-Q-TOF-MS was used to identify the effect of ACT on the cell metabolism. PCA (Fig. 5a) and PLS-DA (Fig. 5b) illustrated that the control, LPS, and ACT groups could be well distinguished based on intracellular metabolites. The levels of various metabolites in LPS-induced BV-2 cells were changed after ACT treatment (Fig. 5c). Compared with the control group, there were 11 metabolites changed significantly in LPS group (Table S2, see Additional file 2). Whereas 14 metabolites were distinctly altered after the treatment of ACT (Table S3, see Additional file 3), involving 11 metabolic pathways (Fig. 5d). The effect of ACT mainly consisted of regulating amino acid metabolism (phenylalanine, tyrosine and tryptophan biosynthesis, D-Glutamine and D-glutamate metabolism, Arg biosynthesis, phenylalanine metabolism), nucleotide metabolism (purine metabolism, pyrimidine metabolism), energy metabolism (nitrogen metabolism), as well as metabolism of cofactors and vitamins (pantothenate and CoA biosynthesis). Interestingly, the metabolic pathways obtained by metabolome were consistent with RNA-seq, including Arg biosynthesis as well as pantothenate and CoA biosynthesis. The above showed that ACT could regulate Arg biosynthesis as well as pantothenate and CoA biosynthesis in LPS-stimulated BV-2 cells. 


\section{Act Mitigated Lps-induced Bv-2 Mitochondrial Dysfunction}

Mitochondria are at the core of metabolic pathways. Evidence is evolving that mitochondria are key players in microglial M1/M2 polarization. Previous research demonstrated that LPS could cause mitochondrial dysfunction. An overview of the mitochondria status in morphology and cell distribution were judged by TEM. After LPS stimulation, BV-2 cells showed nucleus chromatin condensation, decreased cytoplasm, as well as fewer mitochondria (Fig. 6a). In addition, the mitochondrial cristae of the LPS group were disarranged or even disappeared, exhibiting partial cristolysis, reduced size, and roundshaped morphology. Interestingly, ACT treatment could alleviate LPS-induced morphological changes on mitochondria. By turns, we examined the mitochondria function of LPS-treated BV-2 cells, including MMP, and mitochondrial ATP production. The MMP (Fig. 6b, 6c) and ATP production in mitochondria (Fig. $6 \mathrm{~d}$ ) were significantly improved in cells treated with ACT compared to those in the LPS group. Mitochondrial dysfunction may be related to the increased level of ROS in the cell. Flow cytometry analysis revealed that the contents of ROS were overloading in the LPS group (Fig. 6e, 6f). Fortunately, ACT eliminated excessive ROS. It suggests that ACT may restore mitochondria function by clearing ROS.

\section{ACT restored mitochondria function through the upregulation of PGC-1a and UCP-2}

Peroxisome proliferative activated receptor-y co-activator-1a (PGC-1a) plays an important role in mitochondrial biogenesis ${ }^{[15]}$. The stimulation of LPS decreased the expression of PGC-1a, exhibiting mitochondria dysfunction. Remarkably, PGC-1a gene mRNA and protein expression were significantly reversed by ACT treatment in LPS-treated BV-2 cells (Fig. 7a).

The mitochondrial uncoupling protein-2 (UCP-2) also was known to regulate mitochondrial functions. As a downstream protein of PGC-1 $a$, it can control LPS-induced MMP depolarization and ROS production. Recent reports indicate that it is central to the process of microglial activation, with opposite regulation of M1 and M2 polarization ${ }^{[16]}$. Western blot results showed that UCP-2 protein level was decreased after LPS stimulation. The co-treatment of LPS and ACT could upregulate the expression of UCP-2 as compared to the LPS treatment. The mRNA expression level of UCP-2 also showed similar changes (Fig. 7b). Taken together, ACT restored mitochondria function through the upregulation of PGC-1a and UCP-2.

\section{ACT repressed microglia M1 polarization through mitochondrial function recovery via AMPK activation}

AMP-activated protein kinase (AMPK), as the key cellular energy sensor, plays an important role in maintaining cell metabolism balance. At the same time, PGC-1a is a downstream protein of AMPK. Results uncovered that LPS inhibited the activation of AMPK, resulting in cell metabolism disorders and mitochondrial dysfunction. It's noteworthy that ACT could dose-dependently increase the protein expression of p-AMPK (Fig. 8a). It's suggested that ACT might increase the expression of PGC-1a and restore mitochondrial function by activating AMPK signalling pathway.

In this study, to investigate whether the activation of AMPK contributed to the regulation effect of ACT on M1/M2 polarization, compound C (CC) was employed to inhibit the effect of AMPK. In contrast to the 
downregulated NO level in ACT treatment group, CC partly blocked the effect of ACT on NO level (Fig. 8d). Based on these results, ACT could regulate M1/M2 polarization of BV-2 cells by the activation of AMPK.

\section{ACT bond to and inhibited NF-KB as well as activated AMPKa}

Molecular docking was applied to confirm whether ACT binds to the NF-KB and AMPKa proteins. Findings demonstrated that the binding energy of ACT and NF-KB was $-8.4 \mathrm{kcal} / \mathrm{mol}$, which of ACT and AMPKa was $-10.8 \mathrm{kcal} / \mathrm{mol}$. Significant affinities verified that ACT directly bound to NF-KB and AMPKa (Fig. 9). Subsequently, the possible binding modes and interactions within the amino acid pocket were further explored, including Phe A146, Pro A147, Asn A240, Leu A236, Arg A232, His A183, Arg A239, Glu A179, Cys A149, and Tyr A227 of NF-kB (Fig. 9c) as well as Phe A213, Gly A481, Ala A370, Leu A482, Arg A212, Ile A369, Arg A106, Phe A215, Phe A108, Thr A309, Ser A119, and Thr A224 of AMPKa (Fig. 9f). These results indicated that ACT might directly affect NF-KB and AMPKa to attenuate BV-2 microglia M1 polarization and promoted the M2 phenotype.

\section{Discussion}

$A D$ is a progressive neuronal and cognitional dysfunction disease, with complex dysregulated mechanisms ${ }^{[17]}$. Accumulating evidence has demonstrated a significant association between microgliadriven inflammation in the brain. It seems to play a critical role in the progression of AD. Microglia are macrophages in brain ${ }^{[18]}$. It could be activated to a classically M1 inflammatory phenotype, characterized by enhanced secretion of proinflammatory cytokines ${ }^{[4]}$. Excessive $M 1$ activation could accelerate neuron damage and neurodegeneration, even exacerbate to $A D^{[19]}$. Thus, it's imperative to seek new therapeutic approaches aimed at controlling microglia polarization points that could provide adaptive benefits.

Our previous work has verified that ACT had significant effects of improving the learning and memory ability, and protecting the neurons in rat ${ }^{[20]}$. Consistently, the present study also proved that ACT could relieve $\mathrm{AlCl}_{3}$-induced dyskinesia and cholinergic system disorder in zebrafish. Excitedly, ACT presented remarkable anti-inflammatory activities in LPS-induced BV-2 cells. The transcriptomic profile confirmed the significant changes in LPS-induced cells compared with control cells, as well as ACT-treated cells compared with LPS-induced.

ACT suppressed M1 polarization by inhibiting the NK-кB pathway. Except for the NF-KB pathway, RNA-seq also discovered that ACT treatment could affect arginine biosynthesis as well as pantothenate and CoA biosynthesis. Interestingly, the two metabolic pathways were further confirmed by HPLC-Q-TOF-MS analysis. It's widely reported that iNOS could metabolize Arg to NO and citrulline whereas Arg-1 could hydrolyze Arg to ornithine and urea, associated with neuron repair ${ }^{[21]}$. LPS stimulation led to the upregulation of iNOS (Fig. 3a) and downregulation of Arg-1 (Fig. 3b), resulting in increased NO level (Fig. 2f). The data uncovered that ACT alleviated the increased NO level through arginine biosynthesis. 
Pantothenic acid (PA) is the primary substrate for pantothenate kinase ${ }^{[22]}$, as a rate-limiting metabolite in CoA biosynthesis. PA is the obligate precursor of acetyl-CoA, which of particular importance for cholinergic neurons ${ }^{[23]}$ and participate in tricarboxylic acid cycle (TCA cycle) ${ }^{[24]}$. Recent study showed that elevated concentration of CoA would lead to altered mitochondrial morphology, and lower ATP content ${ }^{[22]}$. LPS-induced BV-2 cells exhibited a decrease in the number of mitochondria and a change of mitochondrial shape. After induced by LPS, the production of ROS increased in BV-2 cells. Then the overladen ROS caused membrane phospholipid to be attacked by free radical ${ }^{[25]}$. It led to the loss of MMP, in turn mitochondrial dysfunction and ATP depletion. It was outstanding that ACT treatment mitigated the decrease of MMP and ATP content. These data suggested that ACT induced mitochondrial dysfunction by regulating pantothenate and CoA biosynthesis.

It has been extensively reported that microglia polarization is closely associated with cell metabolism ${ }^{[14]}$. Particularly, as the metabolic hub, mitochondria play remarkable roles in regulating cell metabolism. Recently, mitochondria have been positioned as a key determinant point in microglia polarization ${ }^{[26]}$. To better understand the mechanism of ACT, we judged the functional axis of mitochondria by western blot analysis. It revealed that ACT induced mitochondrial dysfunction by the activation of AMPKa/PGC1/UCP-2 axis.

PGC-1a and UCP-2 are both related to mitochondrial biogenesis ${ }^{[27,28]}$, and they can be thought as the master regulator of ROS ${ }^{[29]}$. Reports indicate that PGC-1a-mediated mitochondrial biogenesis and reduction of ROS are dependent on induction of UCP-2 ${ }^{[27-29]}$. Due to overloading ROS, the expression of PGC-1a and UCP-2 was down-regulated in LPS-induced BV-2 cells. It suggested that ACT could eliminate excessive ROS through PGC-1 $a$ and UCP-2, thus restoring the mitochondrial function. According to the literature, the alteration of PGC-1a in BV-2 cells could contribute to regulating polarization. Interestingly, previous report has found that increased PGC-1 a expression inhibited the NF-KB activity in LPS-induced $\mathrm{BV}-2$ cells ${ }^{[30]}$. It qualified the relationship between PGC-1a and NF-KB in our study.

The expression of PGC-1a is affected by upstream pathway proteins, such as AMPK. AMPK is the key protein for the maintenance of cellular homeostasis ${ }^{[31]}$, playing various roles in promoting M2 polarization of microglia ${ }^{[32]}$. It modulates metabolic pathways in cells ${ }^{[33]}$. We found that ACT promoted the activation of AMPK. At the same time, the application of compound C (AMPK inhibitor) blocked the effect of ACT on attenuating LPS-induced NO excess. Therefore, ACT also suppressed LPS-stimulated M1 polarization via AMPK signaling pathway.

It's the first time to report the mechanism of ACT on regulating microglia polarization (Fig. 10). The data supported that ACT could be developed as a therapeutic agent for neurodegenerative disease associated with neuroinflammation, such as AD. Especially, we linked the microglia polarization to cell metabolism, explaining the effect of ACT through the alteration of mitochondria function. The identification of this metabolic axis, the targeting of this as a unique entity, could allow much better therapeutic approaches against microglia M1 polarization, particularly in AD. 


\section{Abbreviations}

$A D$

Alzheimer's disease; ACT:Acteoside; RNA-Seq:RNA-Sequencing; dpf:days post-fertilization; DPZ:donepezil hydrochloride; AS:average speed; $\triangle S$ :speed change; DRR:dyskinesia recovery rate; RE:response efficiency; ELISA:enzyme-linked immunosorbent assay; LPS:Lipopolysaccharide; CCK-8:Cell Counting Kit-8; AChE:acetylcholinesterase; ChAT:choline acetyltransferase; NO:Nitric oxide; QC:quality control; QTOF:Quadrupole Time-of-Flight; PCA:Principal components analysis; PLS-DA:partial least-squares discriminant analysis; MMP:mitochondrial membrane potential; ROS:reactive oxygen species; ATP:mitochondrial adenosine 5'-triphosphate; TEM:Transmission electron microscopy; qRTPCR:Quantitative real time polymerase chain reaction; iNOS:nitric oxide synthase; DEGs:differentially expressed genes; GO:Gene ontology; KEGG:Kyoto Encyclopedia of Genes and Genomes; Arg:arginine; GAPDH:Glyceraldehyde-3-phosphate dehydrogenase; PGC-1a:peroxisome proliferative activated receptory co-activator-1a; UCP-2:mitochondrial uncoupling protein-2; AMPK:AMP-activated protein kinase; CC:compound C; PA:Pantothenic acid.

\section{Declarations}

\section{Ethics approval and consent to participate}

All zebrafish experiments were carried out under the supervision of the Animal Ethics Committee of China Pharmaceutical University.

\section{Consent for publication}

Not applicable.

\section{Availability of data and materials}

The datasets used and/or analyzed during the current study are available from the corresponding author on reasonable request.

\section{Competing interests}

The authors declare that they have no competing interests.

\section{Funding}

This work was supported by grants from the National Key R\&D Program of China (2019YFC1711000), National Natural Science Foundation of China (No. 81860773, and No. 81873185), Natural Science Foundation of Jiangsu province (No. BK20181327), and Xinjiang Science Fund for Distinguished Young Scholar Project (No. 2018Q003).

\section{Authors' contributions}


All authors were involved in drafting the article or revising it critically for important intellectual content, and all authors approved the final version to be published. Study conception and design: YQL, FL; acquisition of data: YQL, YC, and SQJ; analysis and interpretation of data: YQL, XLJ, YYS, and SSW. The authors read and approved the final manuscript.

\section{Acknowledgements}

The authors are grateful to Ms. Ping Zhou, Yu-Meng Shen, and Mr. Wei Jiang for their technical assistance.

\section{References}

1. Perea JR, Bolos M, Avila J. Microglia in Alzheimer's Disease in the Context of Tau Pathology. Biomolecules. 2020; 10.

2. Shiao YJ, Su MH, Lin HC, Wu CR. Acteoside and Isoacteoside Protect Amyloid beta Peptide Induced Cytotoxicity, Cognitive Deficit and Neurochemical Disturbances In Vitro and In Vivo. Int J Mol Sci. 2017; 18.

3. Linnerbauer M, Rothhammer V. Protective Functions of Reactive Astrocytes Following Central Nervous System Insult. Front Immunol. 2020;11:573256.

4. Hanslik KL, Ulland TK. The Role of Microglia and the Nlrp3 Inflammasome in Alzheimer's Disease. Front Neurol. 2020;11:570711.

5. Tsukahara T, Haniu H, Uemura T, Matsuda Y. Therapeutic Potential of Porcine Liver Decomposition Product: New Insights and Perspectives for Microglia-Mediated Neuroinflammation in Neurodegenerative Diseases. Biomedicines. 2020; 8.

6. Li L, Wang Y, Wang H, Lv L, Zhu ZY. Metabolic responses of BV-2 cells to puerarin on its polarization using ultra-performance liquid chromatography-mass spectrometry. Biomed Chromatogr. 2020;34:e4796.

7. Agrawal I, Jha S. Mitochondrial Dysfunction and Alzheimer's Disease: Role of Microglia. Front Aging Neurosci. 2020;12:252.

8. Wei W, Lu M, Lan X, Liu N, Wang H, Du J, et al. Neuroprotective effect of Verbascoside on hypoxicischemic brain damage in neonatal rat. Neurosci Lett. 2019;711:134415.

9. Lai X, Xiong Y, Zhou J, Yang F, Peng J, Chen L, et al. Verbascoside attenuates acute inflammatory injury in experimental cerebral hemorrhage by suppressing TLR4. Biochem Biophys Res Commun. 2019;519:721-6.

10. Ji SL, Cao KK, Zhao XX, Kang NX, Zhang Y, Xu QM, et al. Antioxidant activity of phenylethanoid glycosides on glutamate-induced neurotoxicity. Biosci Biotechnol Biochem. 2019;83:2016-26.

11. Chen J, Gao L, Zhang Y, Su Y, Kong Z, Wang D, et al. Acteoside-improved streptozotocin-induced learning and memory impairment by upregulating hippocampal insulin, glucose transport, and energy metabolism. Phytother Res. 2020. 
12. Li W, Deng R, Jing X, Chen J, Yang D, Shen J. Acteoside ameliorates experimental autoimmune encephalomyelitis through inhibiting peroxynitrite-mediated mitophagy activation. Free Radic Biol Med. 2020;146:79-91.

13. Li YQ, Chen Y, Fang JY, Jiang SQ, Li P, Li F. Integrated network pharmacology and zebrafish model to investigate dual-effects components of Cistanche tubulosa for treating both Osteoporosis and Alzheimer's Disease. J Ethnopharmacol. 2020;254:112764.

14. Orihuela R, McPherson CA, Harry GJ. Microglial M1/M2 polarization and metabolic states. Br J Pharmacol. 2016;173:649-65.

15. Bi J, Zhang J, Ren Y, Du Z, Li Q, Wang Y, et al. Irisin alleviates liver ischemia-reperfusion injury by inhibiting excessive mitochondrial fission, promoting mitochondrial biogenesis and decreasing oxidative stress. Redox Biol. 2019;20:296-306.

16. De Simone R, Ajmone-Cat MA, Pandolfi M, Bernardo A, De Nuccio C, Minghetti L, et al. The mitochondrial uncoupling protein-2 is a master regulator of both M1 and M2 microglial responses. $J$ Neurochem. 2015;135:147-56.

17. Fakhri S, Pesce M, Patruno A, Moradi SZ, Iranpanah A, Farzaei MH, et al. Attenuation of Nrf2/Keap1/ARE in Alzheimer's Disease by Plant Secondary Metabolites: A Mechanistic Review. Molecules. 2020; 25.

18. Afridi R, Lee WH, Suk K. Microglia Gone Awry: Linking Immunometabolism to Neurodegeneration. Front Cell Neurosci. 2020;14:246.

19. Bagheri-Mohammadi S. Microglia in Alzheimer's Disease: The Role of Stem Cell-Microglia Interaction in Brain Homeostasis. Neurochem Res. 2020.

20. Chen Y, Li YQ, Fang JY, Li P, Li F. Establishment of the concurrent experimental model of osteoporosis combined with Alzheimer's disease in rat and the dual-effects of echinacoside and acteoside from Cistanche tubulosa. J Ethnopharmacol. 2020;257:112834.

21. Rath M, Muller I, Kropf P, Closs El, Munder M. Metabolism via Arginase or Nitric Oxide Synthase: Two Competing Arginine Pathways in Macrophages. Front Immunol. 2014;5:532.

22. Kumar A, Kumar Y, Sevak JK, Kumar S, Kumar N, Gopinath SD. Metabolomic analysis of primary human skeletal muscle cells during myogenic progression. Sci Rep. 2020;10:11824.

23. Xu J, Patassini S, Begley P, Church S, Waldvogel HJ, Faull RLM, et al. Cerebral deficiency of vitamin B5 (d-pantothenic acid; pantothenate) as a potentially-reversible cause of neurodegeneration and dementia in sporadic Alzheimer's disease. Biochem Biophys Res Commun. 2020;527:676-81.

24. Atamna H. Heme, iron, and the mitochondrial decay of ageing. Ageing Res Rev. 2004;3:303-18.

25. Magnani ND, Marchini T, Calabro V, Alvarez S, Evelson P. Role of Mitochondria in the Redox Signaling Network and Its Outcomes in High Impact Inflammatory Syndromes. Front Endocrinol (Lausanne). 2020;11:568305.

26. Harry GJ, Childers G, Giridharan S, Hernandes IL. An association between mitochondria and microglia effector function. What do we think we know? Neuroimmunol Neuroinflamm. 2020;7:15065. 
27. Uittenbogaard M, Chiaramello A. Mitochondrial biogenesis: a therapeutic target for neurodevelopmental disorders and neurodegenerative diseases. Curr Pharm Des. 2014;20:5574-93.

28. de Oliveira Bristot VJ, de Bem Alves AC, Cardoso LR, da Luz Scheffer D, Aguiar AS. Jr. The Role of PGC-1alpha/UCP2 Signaling in the Beneficial Effects of Physical Exercise on the Brain. Front Neurosci. 2019;13:292.

29. Jamwal S, Blackburn JK, Elsworth JD. PPARgamma/PGC1alpha signaling as a potential therapeutic target for mitochondrial biogenesis in neurodegenerative disorders. Pharmacol Ther. 2020: 107705.

30. Yang X, Xu S, Qian Y, Xiao Q. Resveratrol regulates microglia M1/M2 polarization via PGC-1alpha in conditions of neuroinflammatory injury. Brain Behav Immun. 2017;64:162-72.

31. Qiu WQ, Pan R, Tang Y, Zhou XG, Wu JM, Yu L, et al. Lychee seed polyphenol inhibits Abeta-induced activation of NLRP3 inflammasome via the LRP1/AMPK mediated autophagy induction. Biomed Pharmacother. 2020;130:110575.

32. Chu X, Cao L, Yu Z, Xin D, Li T, Ma W, et al. Hydrogen-rich saline promotes microglia M2 polarization and complement-mediated synapse loss to restore behavioral deficits following hypoxia-ischemic in neonatal mice via AMPK activation. J Neuroinflammation. 2019;16:104.

33. Szewczuk M, Boguszewska K, Kazmierczak-Baranska J, Karwowski BT. The role of AMPK in metabolism and its influence on DNA damage repair. Mol Biol Rep. 2020.

\section{Figures}


a

b
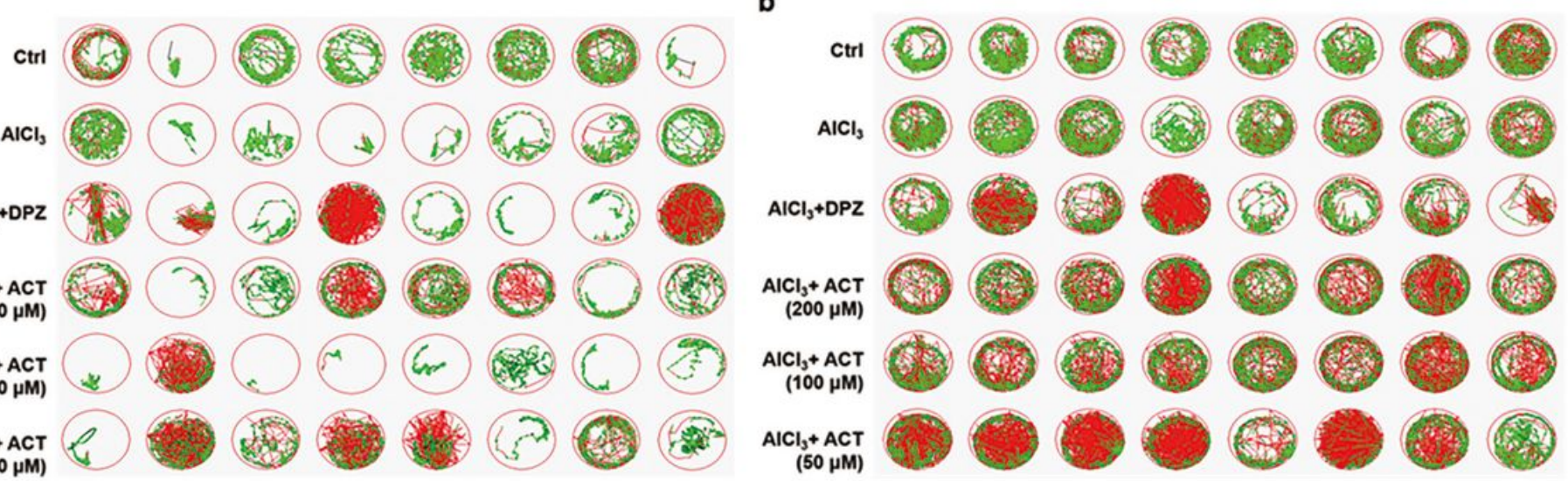

c
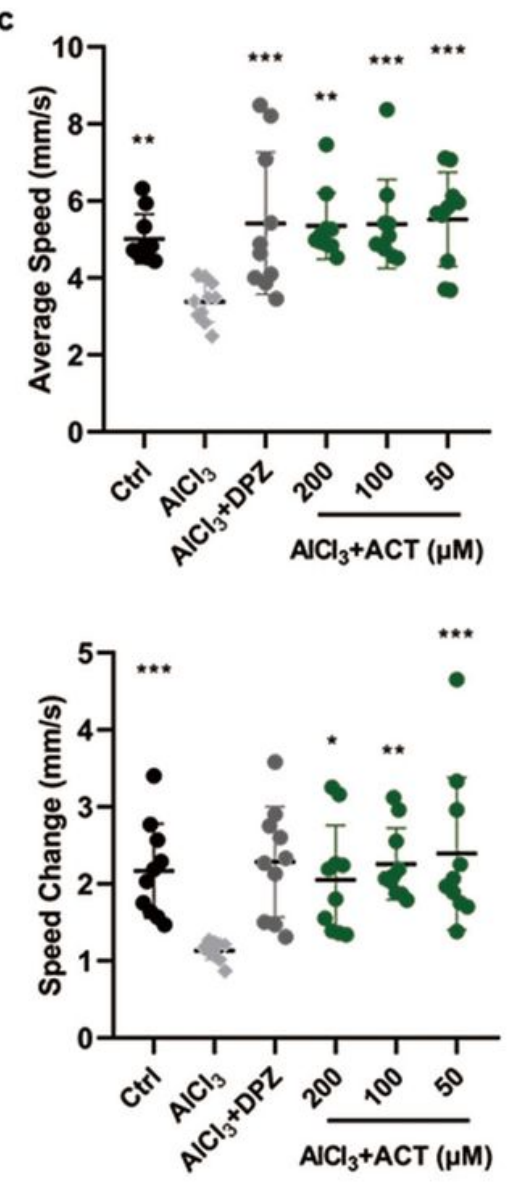

d
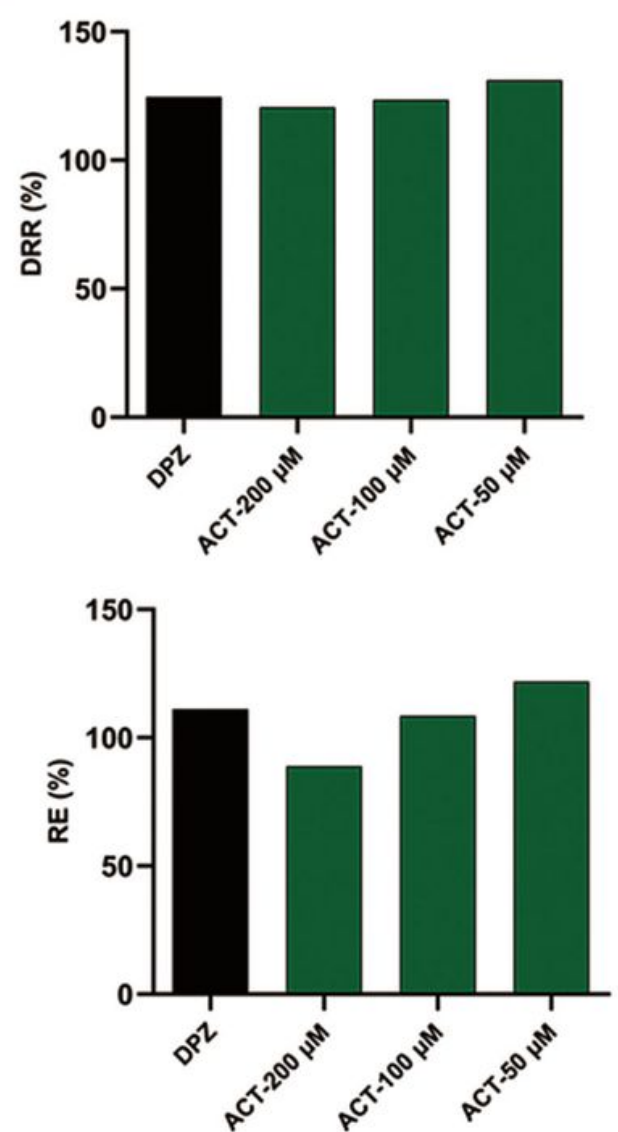

e
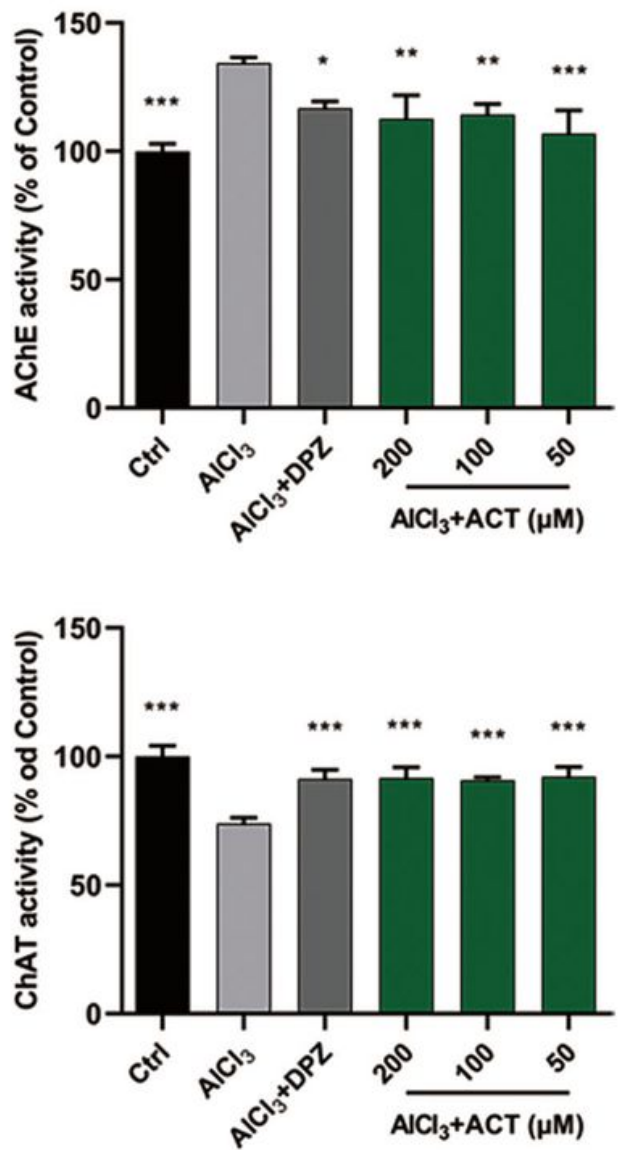

Figure 1

ACT attenuated AICl3-induced AD in zebrafish larvae. (a) The representative swimming trails of zebrafish larvae in light phase. (b) The representative swimming trails of zebrafish larvae in dark phase. (c) The average speed, and speed change in different groups $(n=10)$. (d) DRR (\%), and RE (\%) in the drug

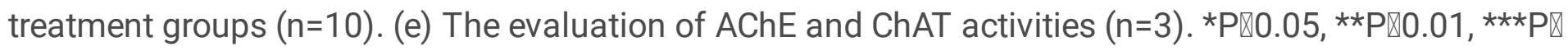
0.005 , versus the $\mathrm{AlCl} 3$ group. DPZ as the positive control. 
a

b
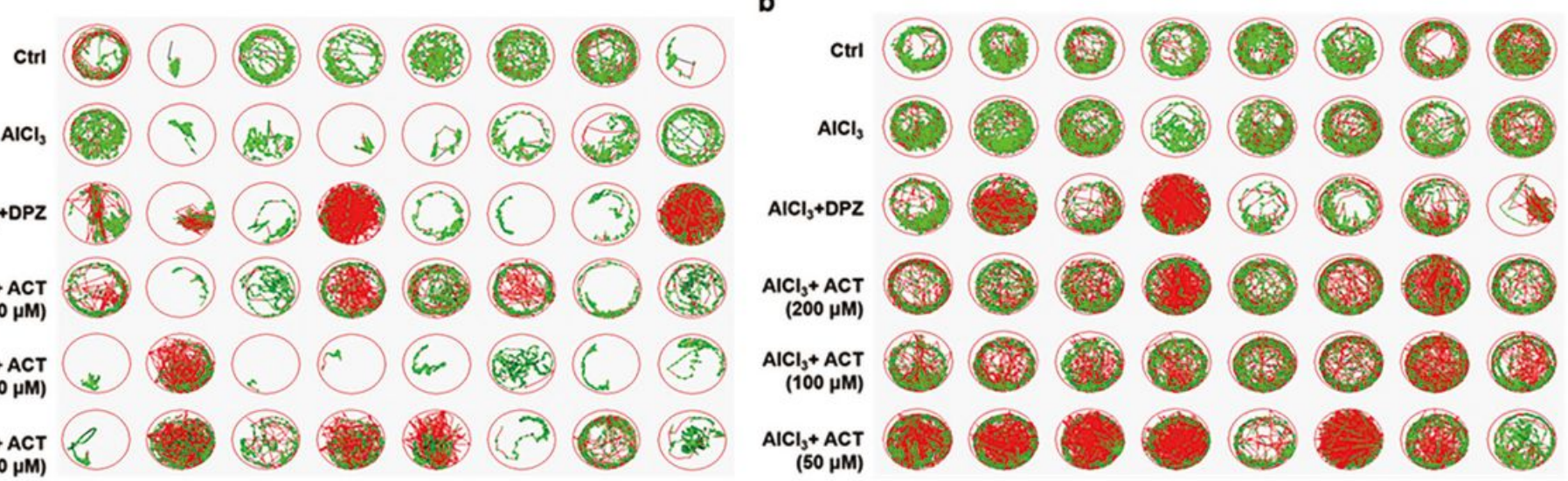

c
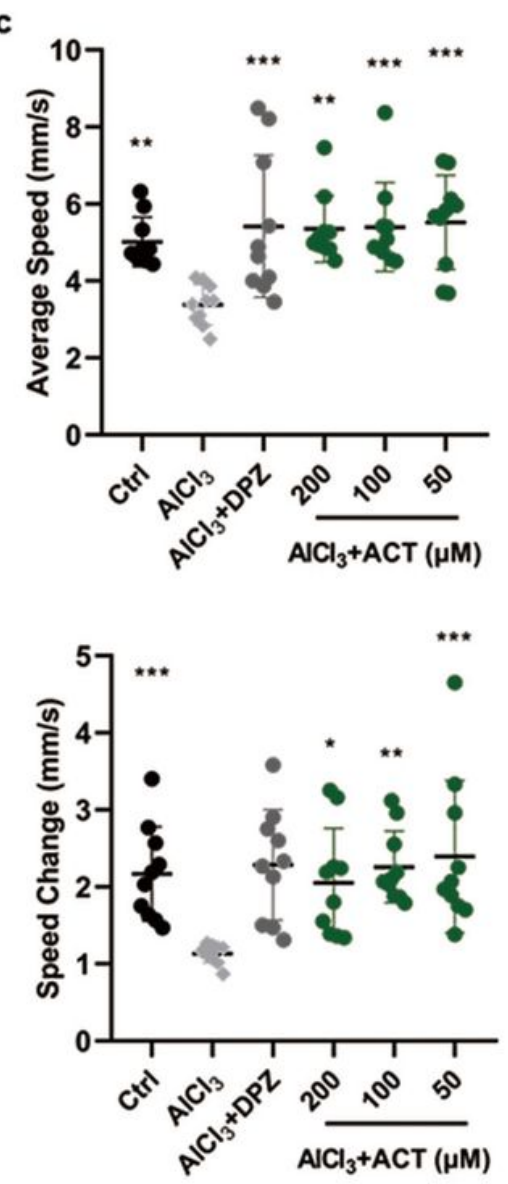

d
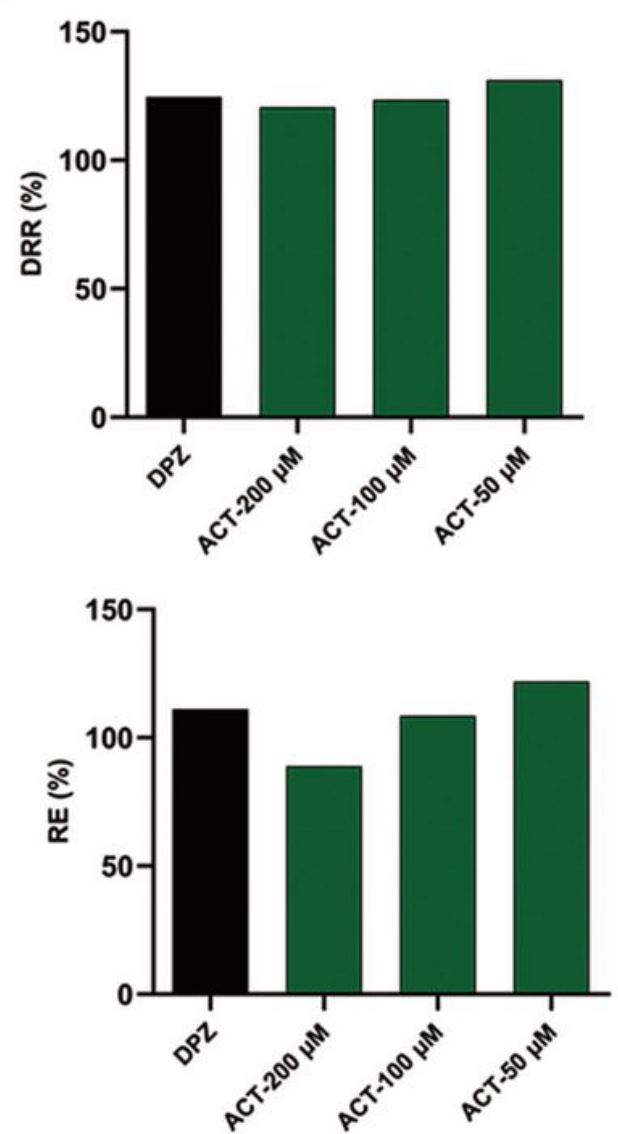

e
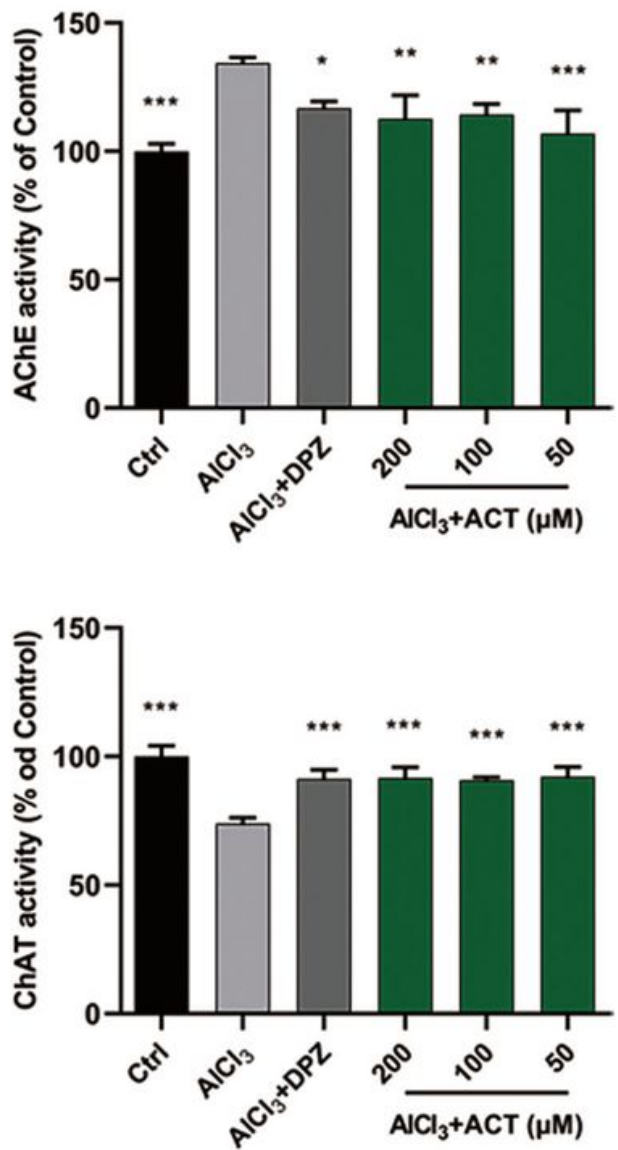

Figure 1

ACT attenuated AICl3-induced AD in zebrafish larvae. (a) The representative swimming trails of zebrafish larvae in light phase. (b) The representative swimming trails of zebrafish larvae in dark phase. (c) The average speed, and speed change in different groups $(n=10)$. (d) DRR (\%), and RE (\%) in the drug

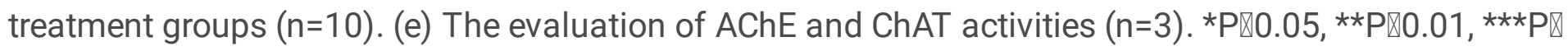
0.005 , versus the $\mathrm{AlCl} 3$ group. DPZ as the positive control. 
a

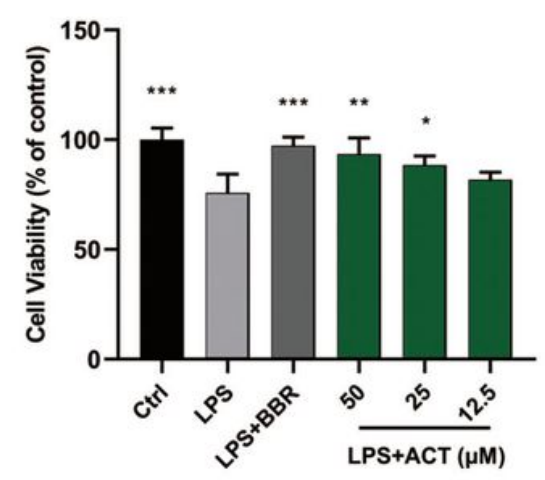

d

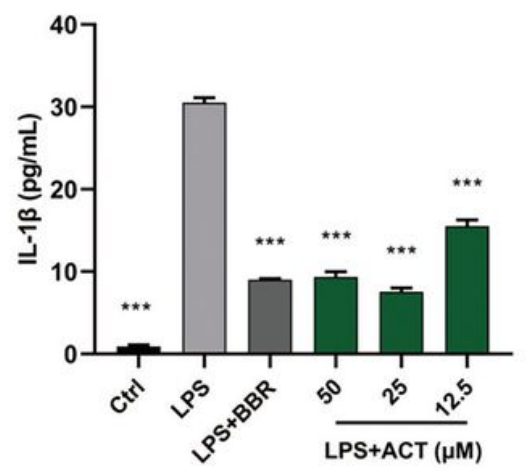

b

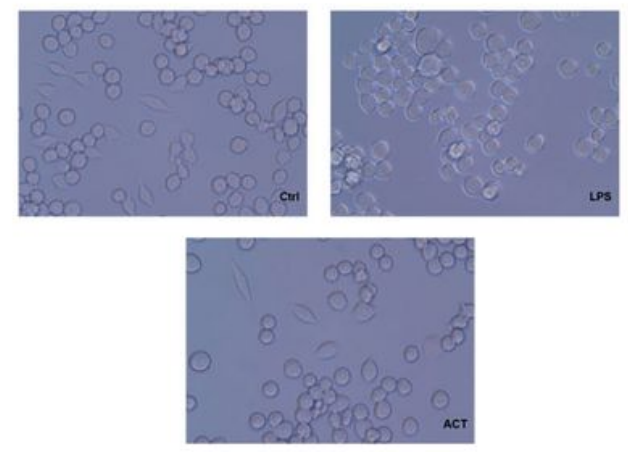

e

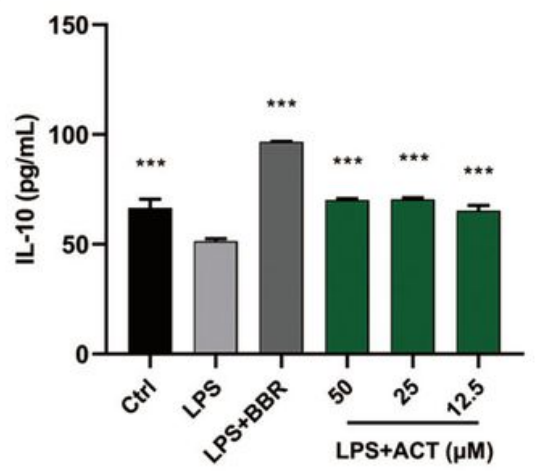

c
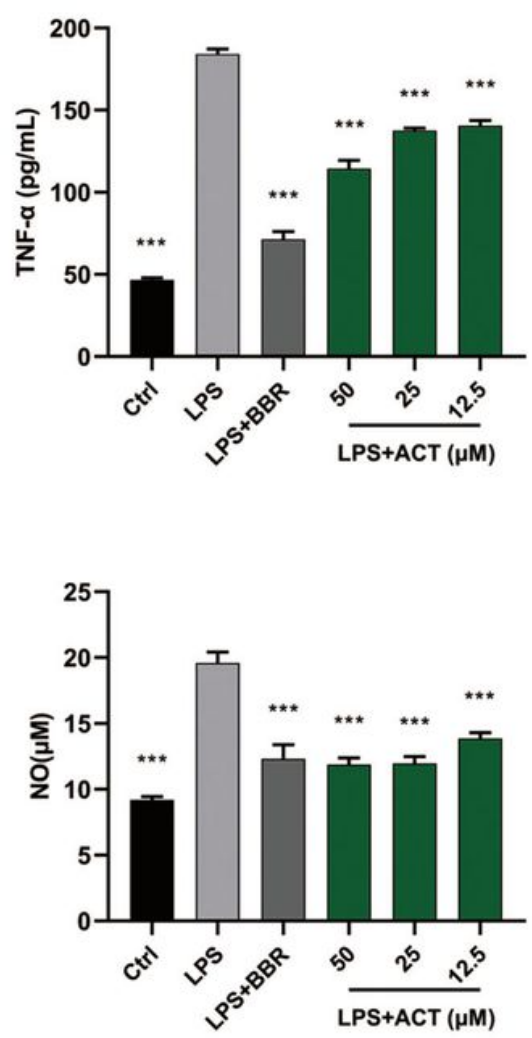

Figure 2

ACT regulated M1/M2 polarization in LPS-stimulated BV-2 cells. (a) Effects of ACT on LPS-induced BV-2 cell viability. (b) Cell morphology was observed with inverted phase contrast microscope $(\times 40)$. The control group exhibited small soma with distal arborization, showing the typical ramified morphology of resting microglia. The LPS group became fewer and shorter branches with a greatly enlarged cell body, the characteristic shapes of activated microglia. The ACT groups showed attenuated LPS-induced morphological changes. (c) Effects of ACT on LPS-induced elevation of cell supernatant TNF-a levels. (d) Effects of ACT on LPS-induced elevation of cell supernatant IL-1 $\beta$ levels. (e) Effects of ACT on LPSinduced decline of cell supernatant IL-10 levels. (f) Effect of ACT on LPS-induced nitric oxide (NO) elevation. ${ }^{*} \otimes 0.05,{ }^{\star} \star * P \otimes 0.005$, versus the LPS group. $n=3$. Berberine (BBR) as a positive control. 
a

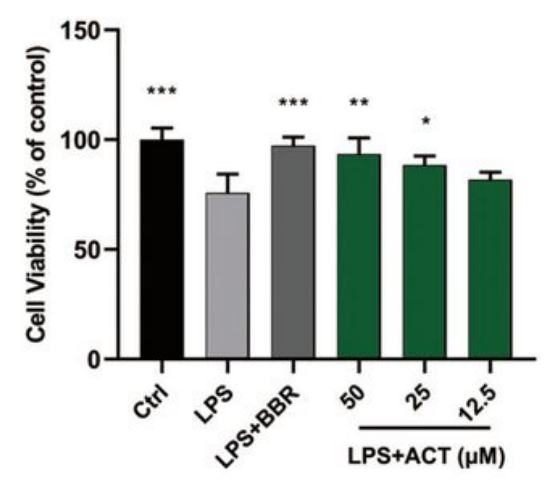

d

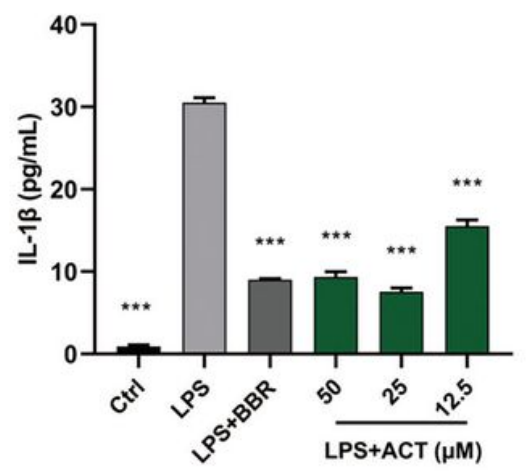

b

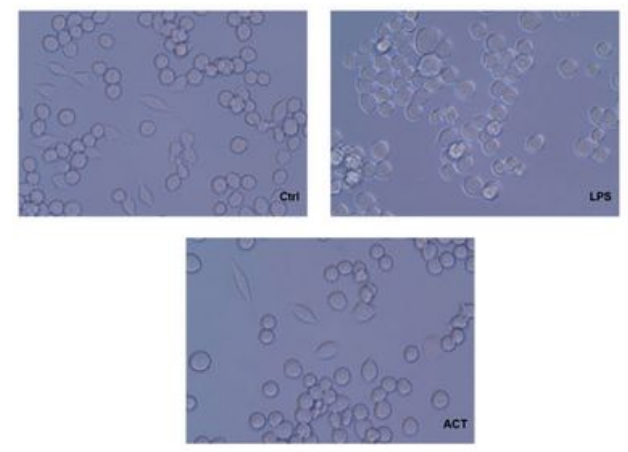

e

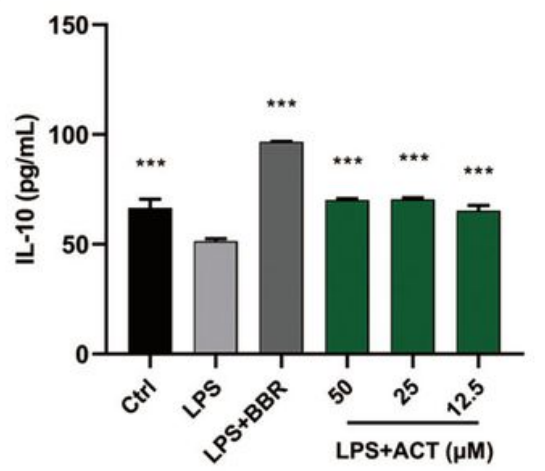

c
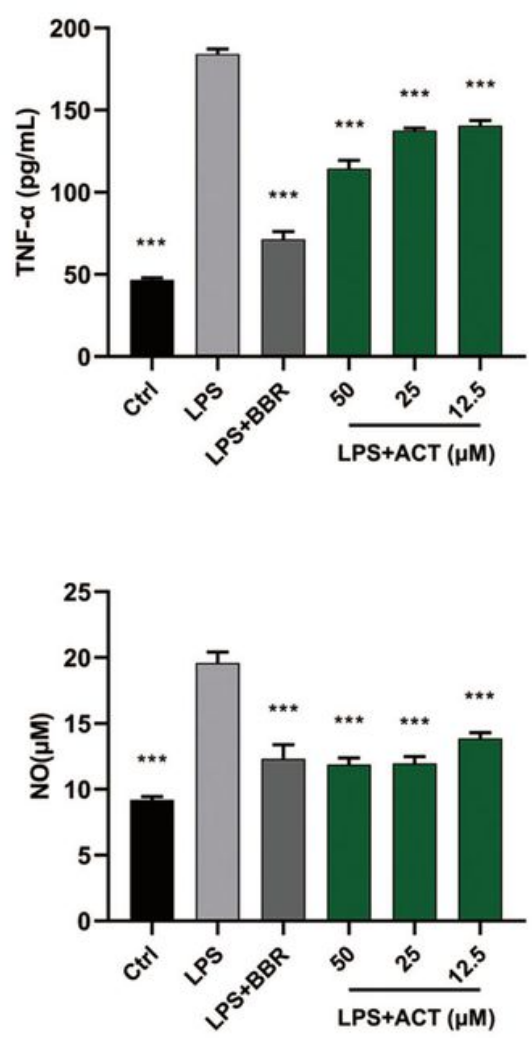

Figure 2

ACT regulated M1/M2 polarization in LPS-stimulated BV-2 cells. (a) Effects of ACT on LPS-induced BV-2 cell viability. (b) Cell morphology was observed with inverted phase contrast microscope $(\times 40)$. The control group exhibited small soma with distal arborization, showing the typical ramified morphology of resting microglia. The LPS group became fewer and shorter branches with a greatly enlarged cell body, the characteristic shapes of activated microglia. The ACT groups showed attenuated LPS-induced morphological changes. (c) Effects of ACT on LPS-induced elevation of cell supernatant TNF-a levels. (d) Effects of ACT on LPS-induced elevation of cell supernatant IL-1 $\beta$ levels. (e) Effects of ACT on LPSinduced decline of cell supernatant IL-10 levels. (f) Effect of ACT on LPS-induced nitric oxide (NO) elevation. ${ }^{*} \otimes 0.05,{ }^{\star} \star * P \otimes 0.005$, versus the LPS group. $n=3$. Berberine (BBR) as a positive control. 
a
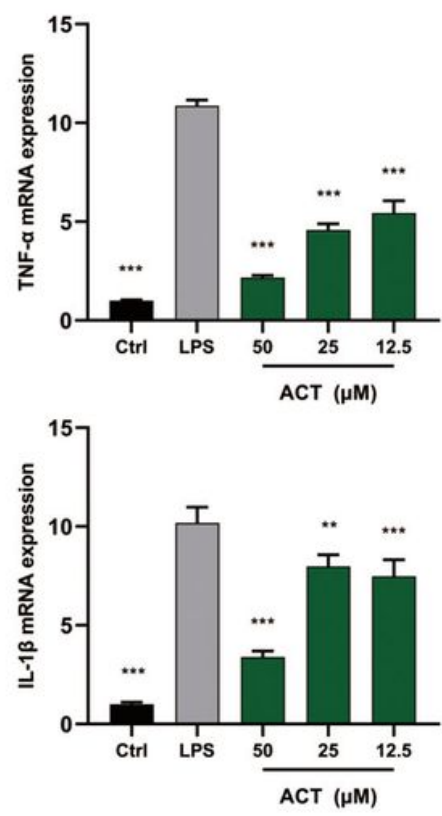
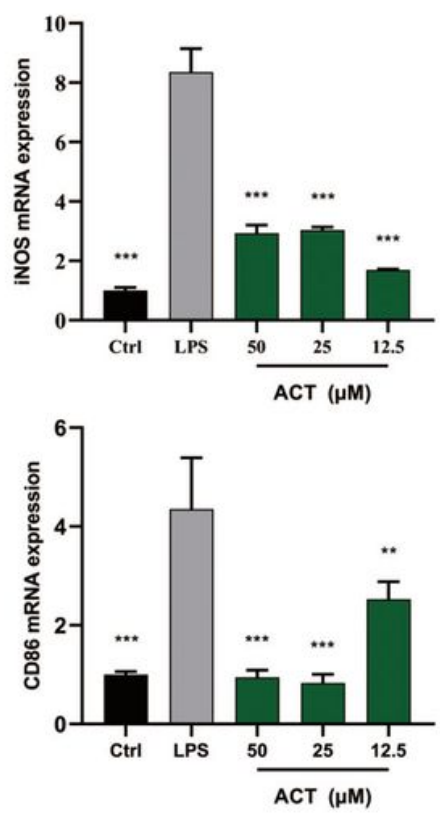

b
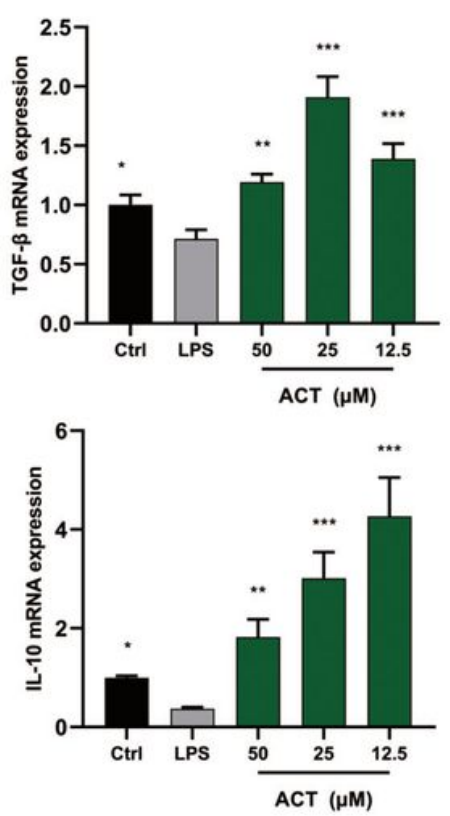
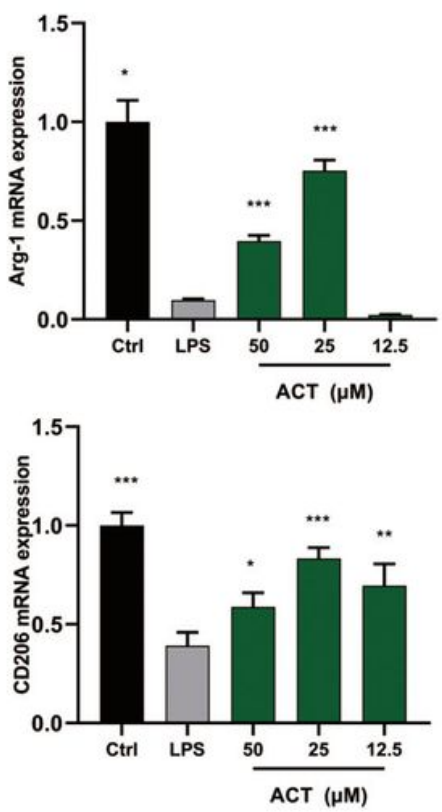

Figure 3

ACT suppressed M1 microglia polarization and promoted the M2 phenotype. (a) Effects of ACT on M1 microglia polarization related markers. (b) Effect of ACT on M2 microglia polarization related markers. *P $\otimes 0.05, * * P \otimes 0.01, * \star * P \otimes 0.005$, versus the LPS group. $\mathrm{n}=3$.
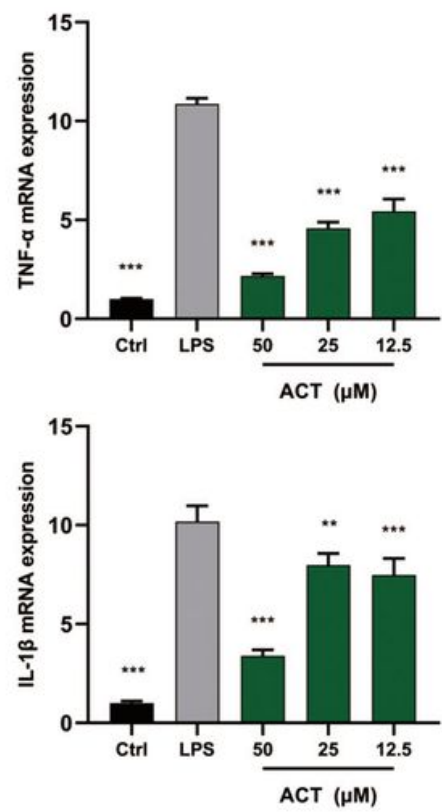
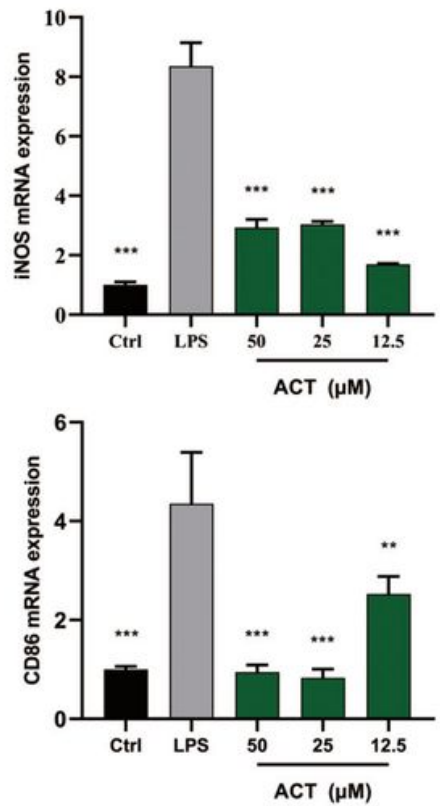
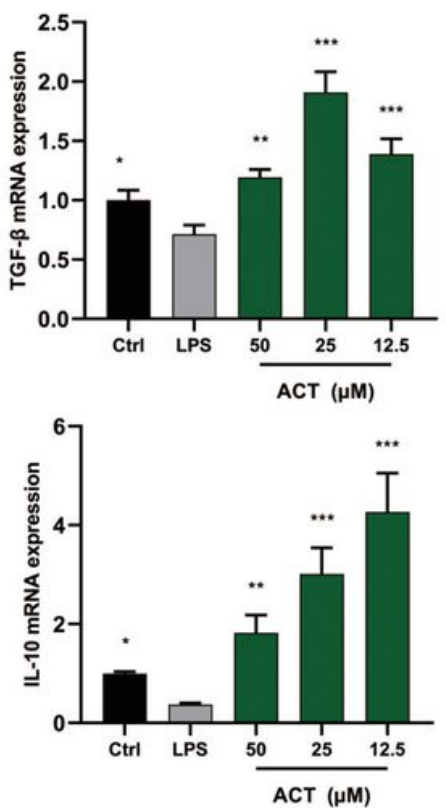
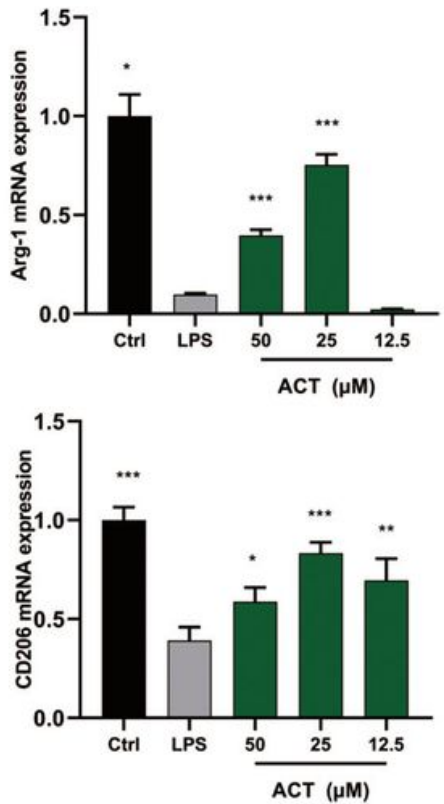

Figure 3

ACT suppressed M1 microglia polarization and promoted the M2 phenotype. (a) Effects of ACT on M1 microglia polarization related markers. (b) Effect of ACT on M2 microglia polarization related markers. *P $\llbracket 0.05, \star * P \otimes 0.01, \star \star \star P \otimes 0.005$, versus the LPS group. $\mathrm{n}=3$. 
a

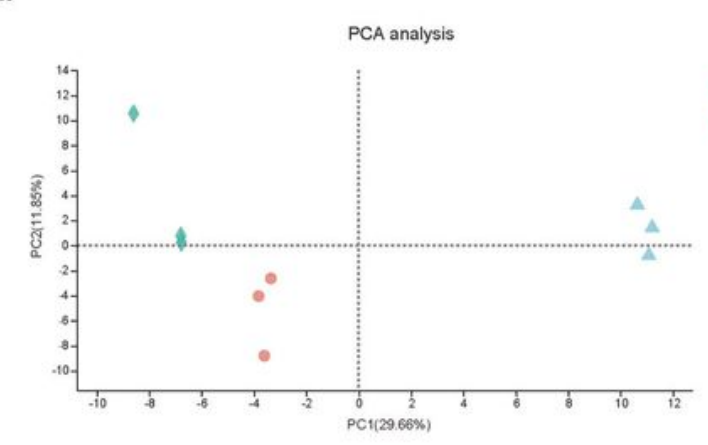

c

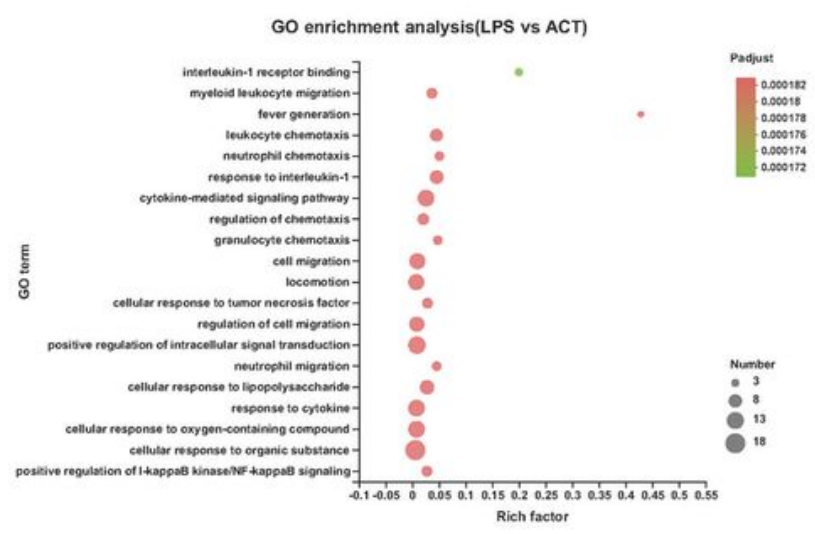

e
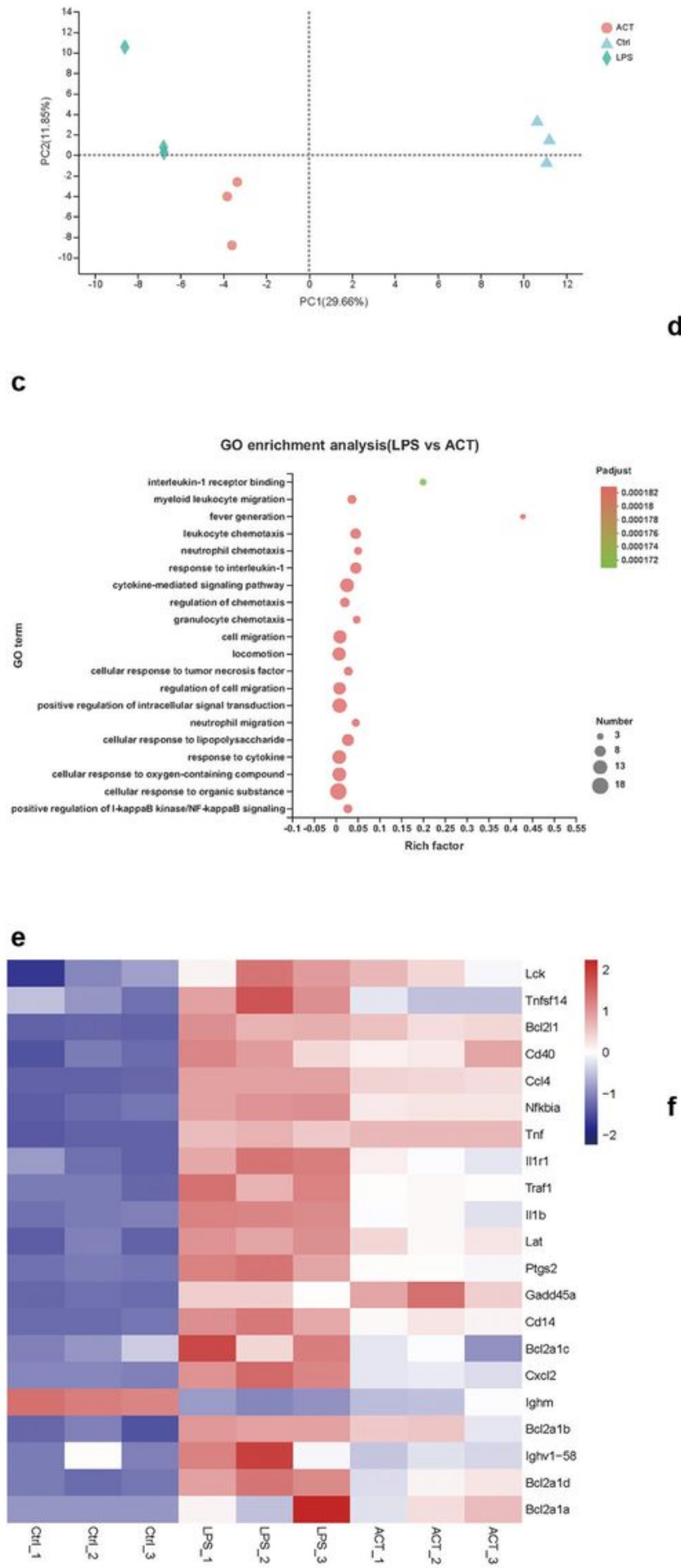

b

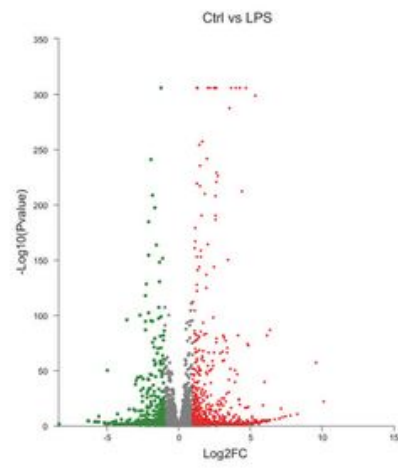

d

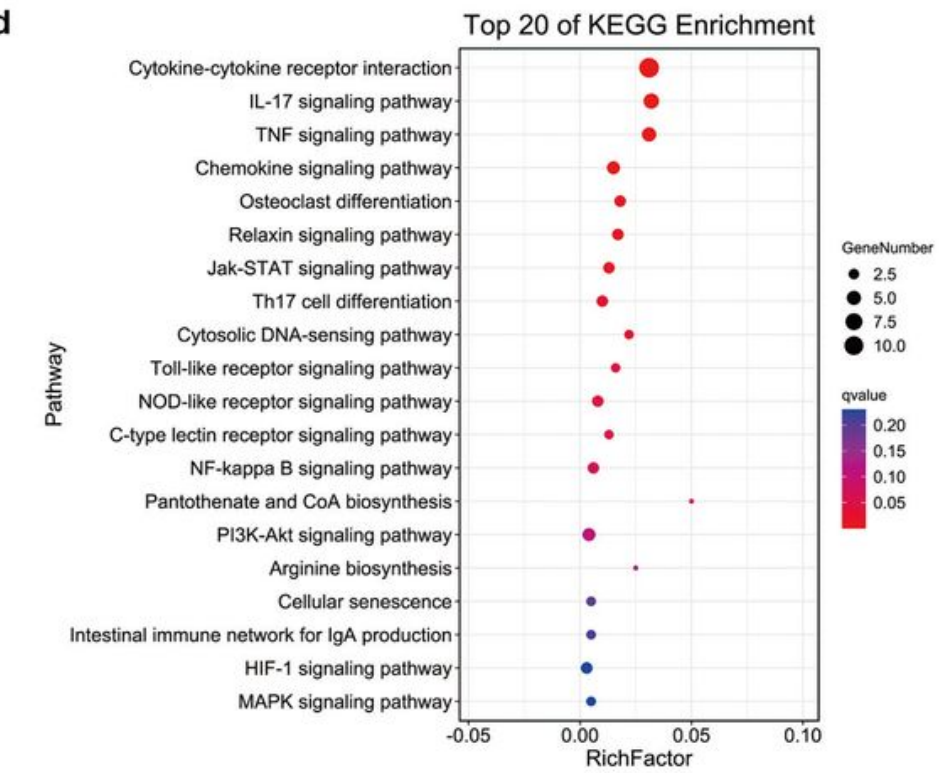

LPS VS LPS+ACT

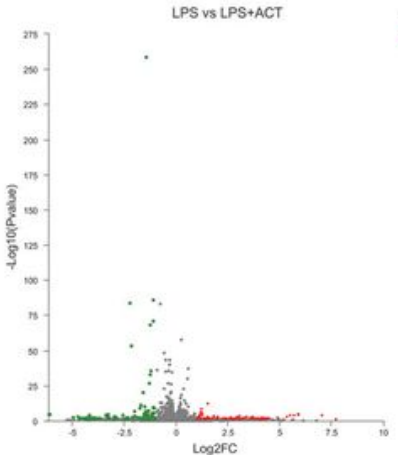

: $\lim _{\operatorname{som}}$ 
by Kyoto Encyclopedia of Genes and Genomes (KEGG) enrichment analysis. (e) Heat map of the differentially expressed genes (DEGs) that were linked NF-KB signalling pathway. (f) ACT distinctly decreased the phosphorylation level of NF-KB $(n=3)$. *P囚0.05, ${ }^{*} P \otimes 0.01$, versus the LPS group.

a

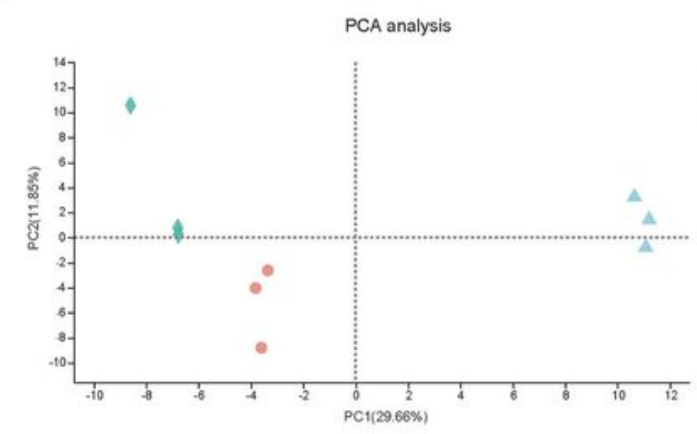

C

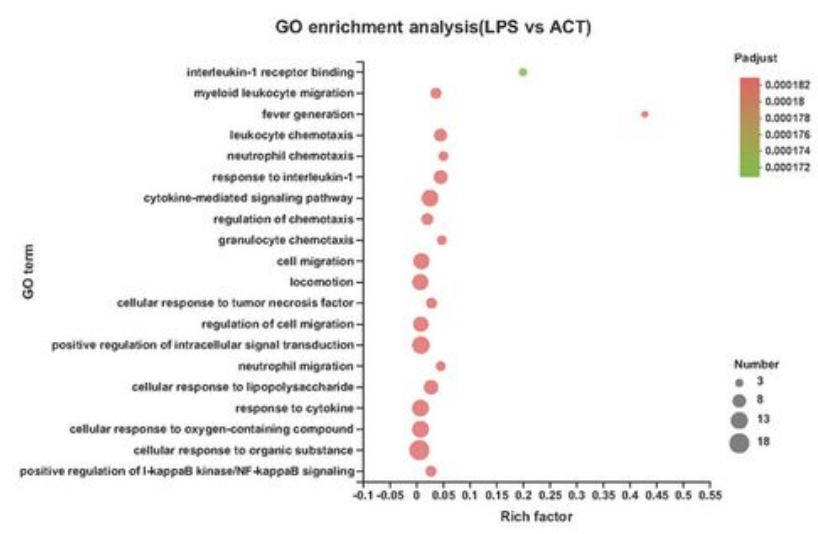

e

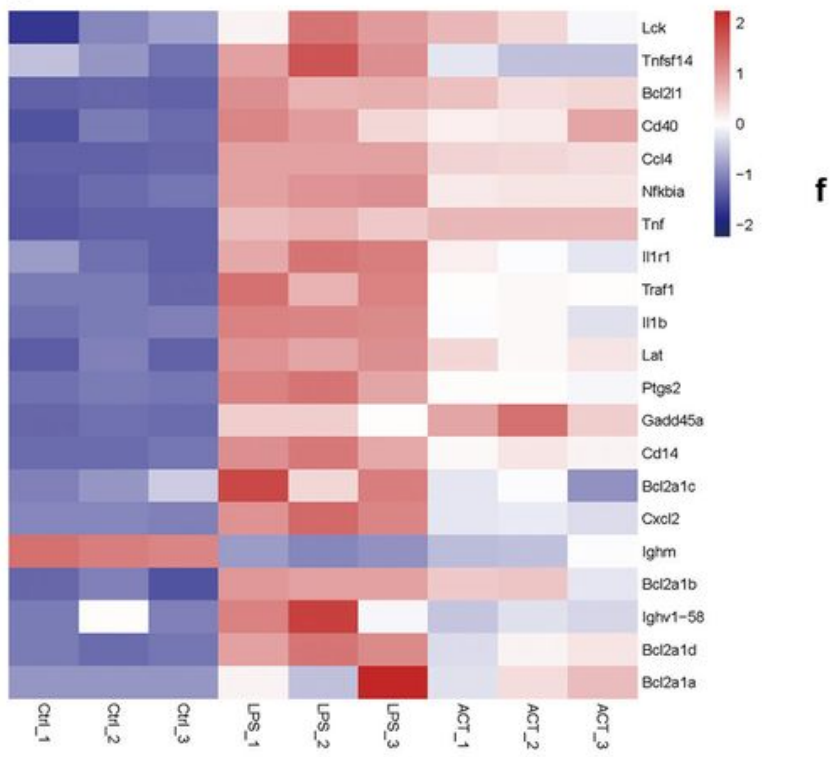

b

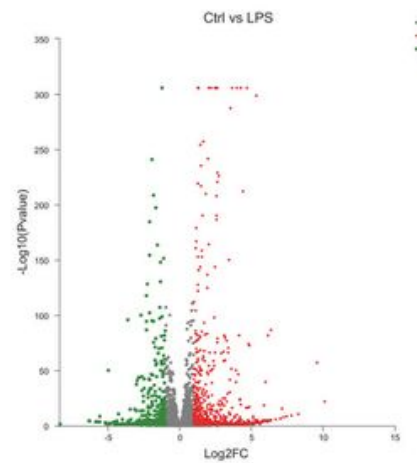

d

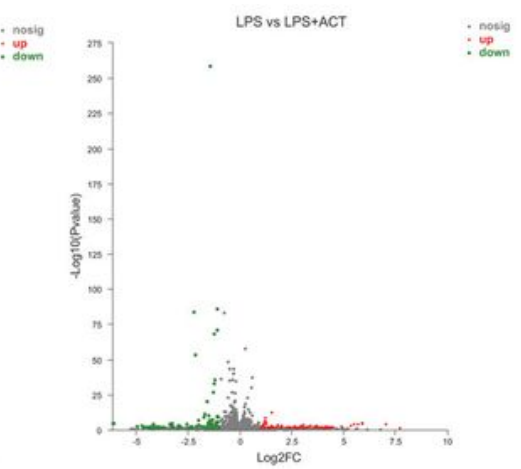

Top 20 of KEGG Enrichment
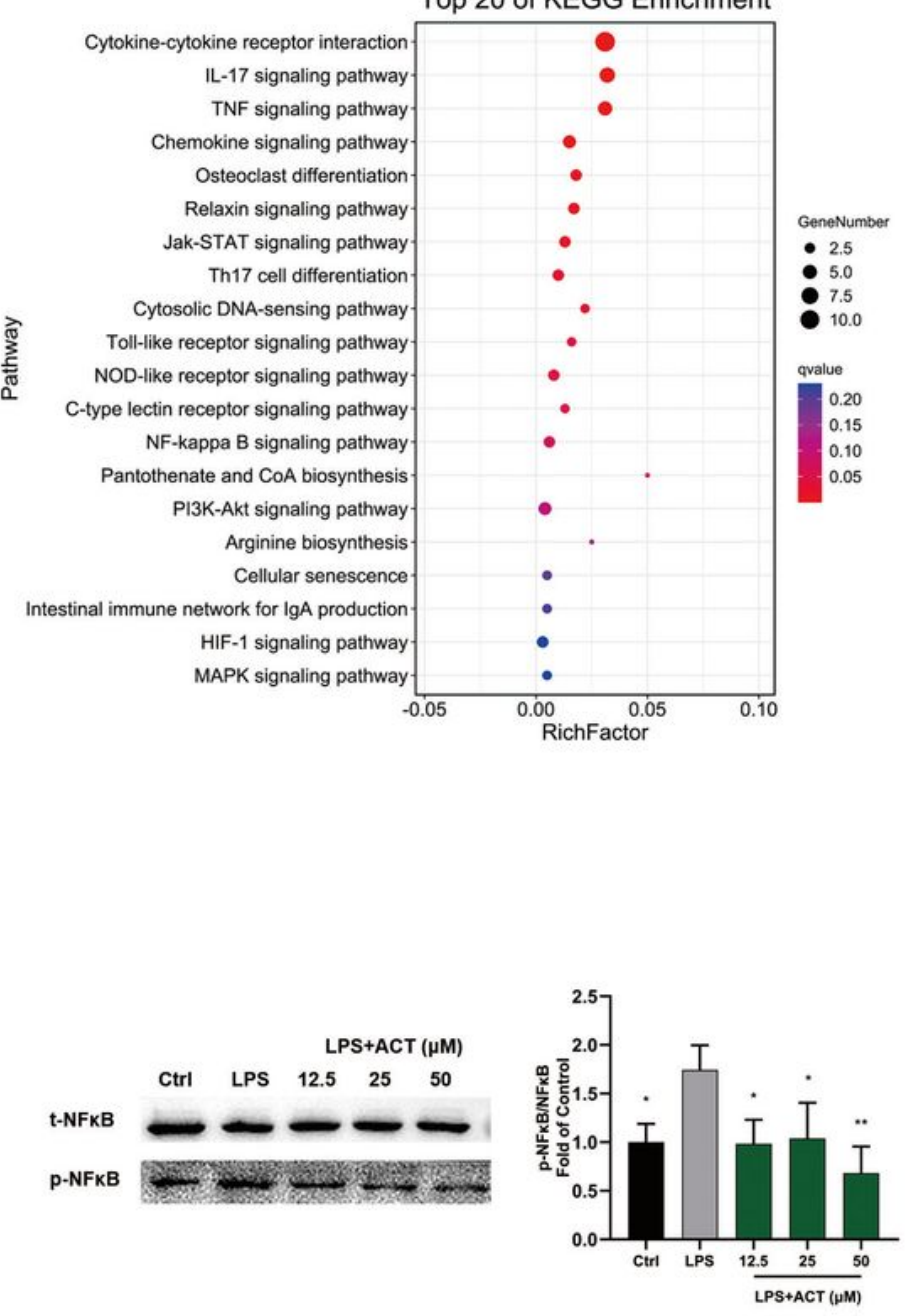

Figure 4

ACT regulated M1/M2 polarization via the inhibition of NF-KB signalling pathway in LPS-induced BV-2 cells. (a) Principal components analysis (PCA) score plot for discriminating the cell metabolome from 
Ctrl, LPS and ACT $(50 \mu \mathrm{M})$ groups from a transcriptomic level $(n=3)$. (b) Volcano plot representing the RNA-seq data of LPS-induced and ACT-treated BV-2 cells. (c) Gene ontology (GO) terms in ACT-treated cells as determined by GO enrichment analysis. (d) Top 20 pathways in ACT-treated cells as determined by Kyoto Encyclopedia of Genes and Genomes (KEGG) enrichment analysis. (e) Heat map of the differentially expressed genes (DEGs) that were linked NF-KB signalling pathway. (f) ACT distinctly decreased the phosphorylation level of NF-KB $(n=3)$. *Pख0.05, **P®0.01, versus the LPS group.

a

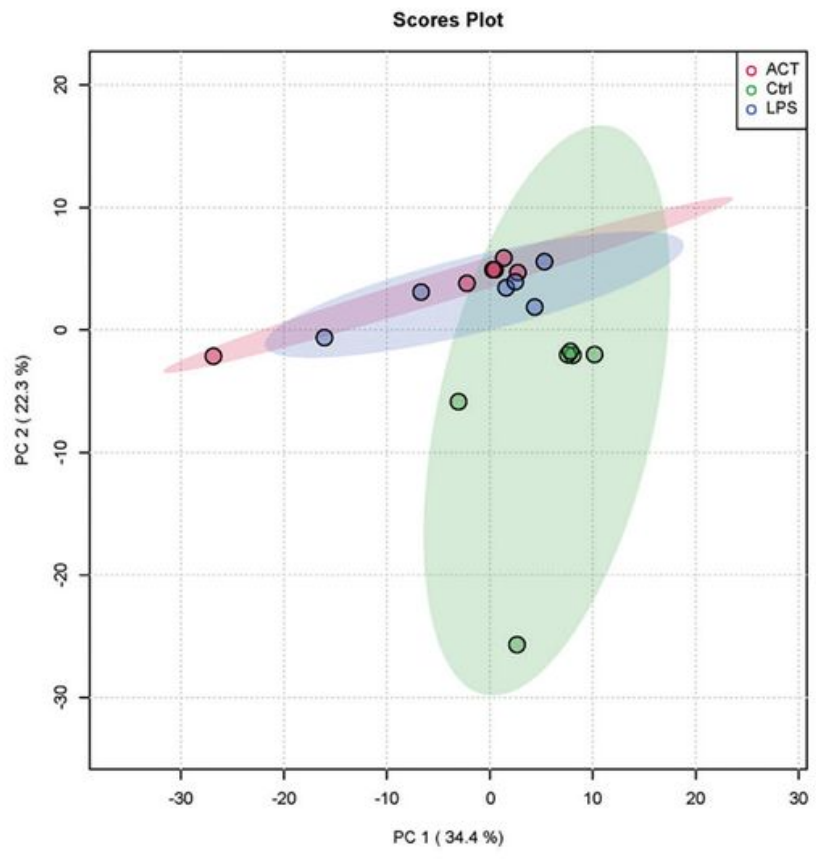

C

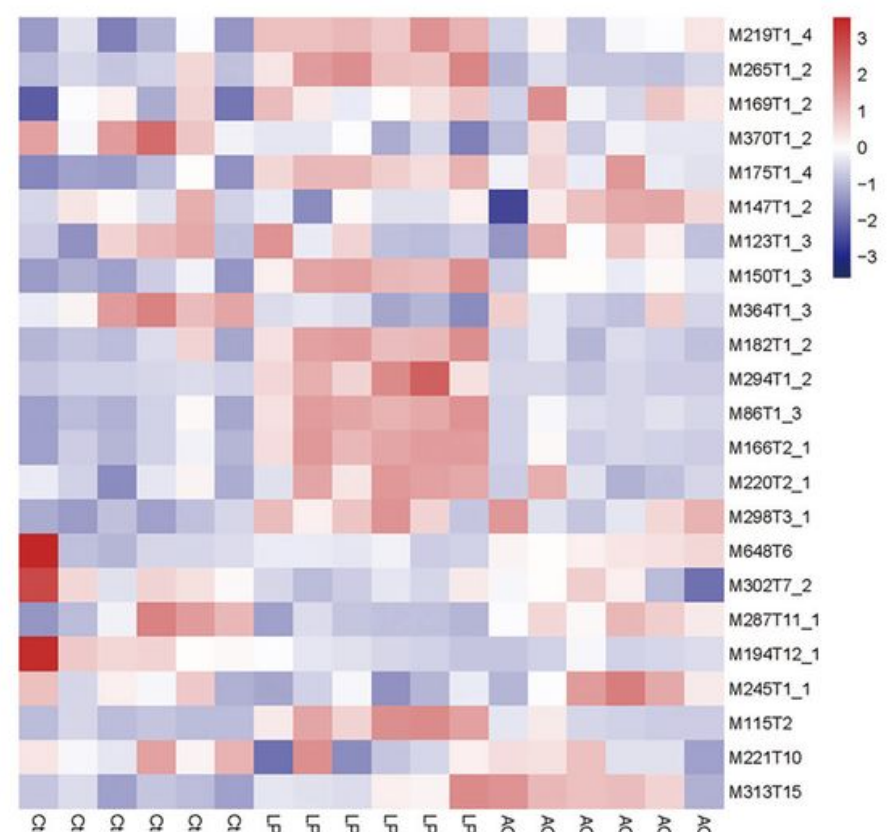

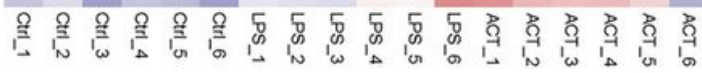

b

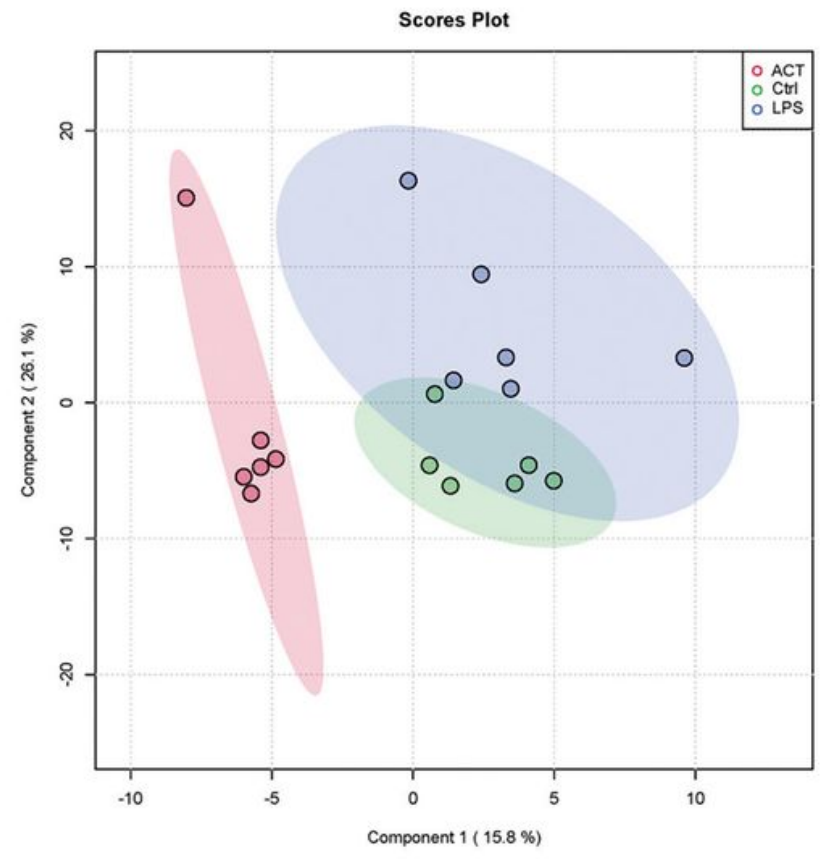

d

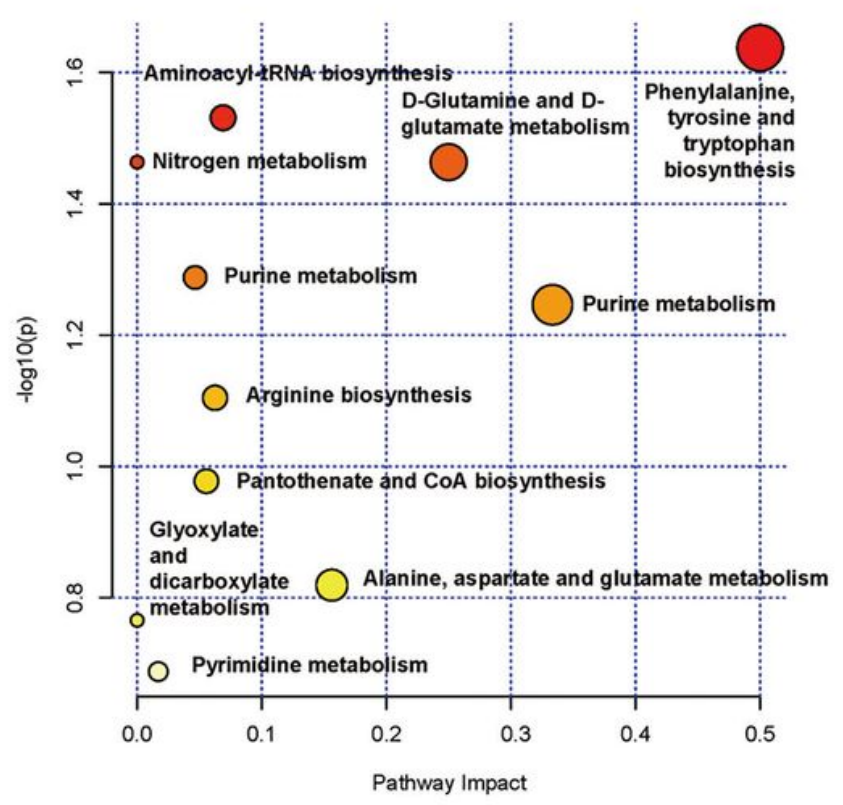

Figure 5 
ACT positively regulated the metabolism shifts of BV-2 cells. (a) Principal components analysis (PCA) score plot and (b) partial least squares-discrimination analysis (PLS-DA) score plot for discriminating the cell metabolome from Ctrl, LPS and ACT (50 $\mu \mathrm{M})$ groups in positive ion mode. (c) Heat map of the differential metabolites that were altered in LPS group compared to Ctrl group and ACT group compared to LPS group $(n=6)$. (d) The disturbed metabolic pathways in ACT group.

a

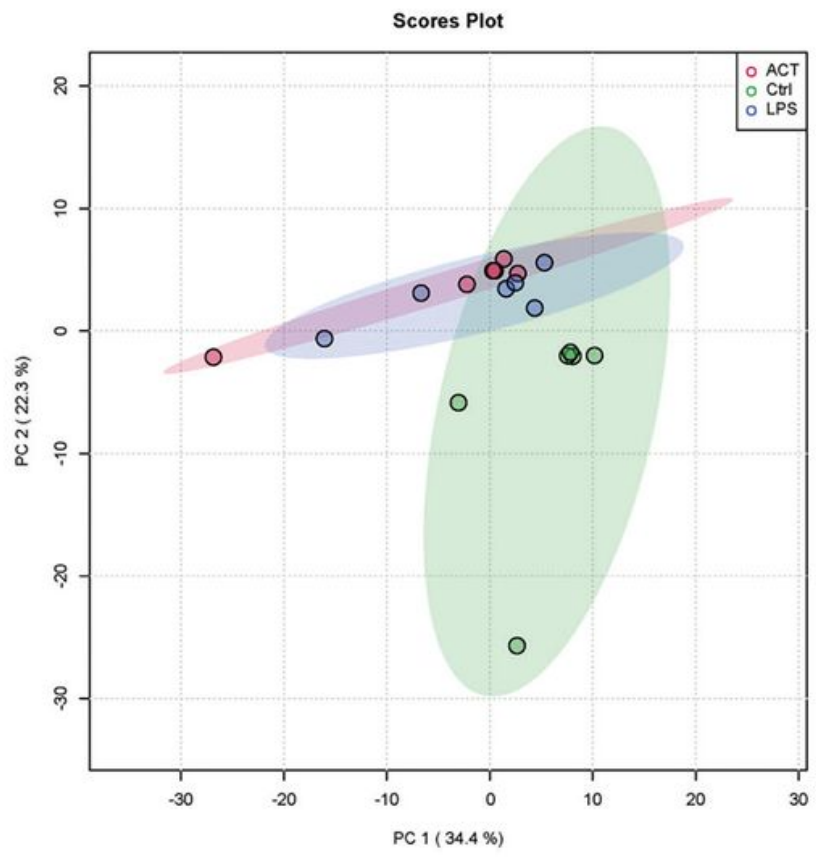

C

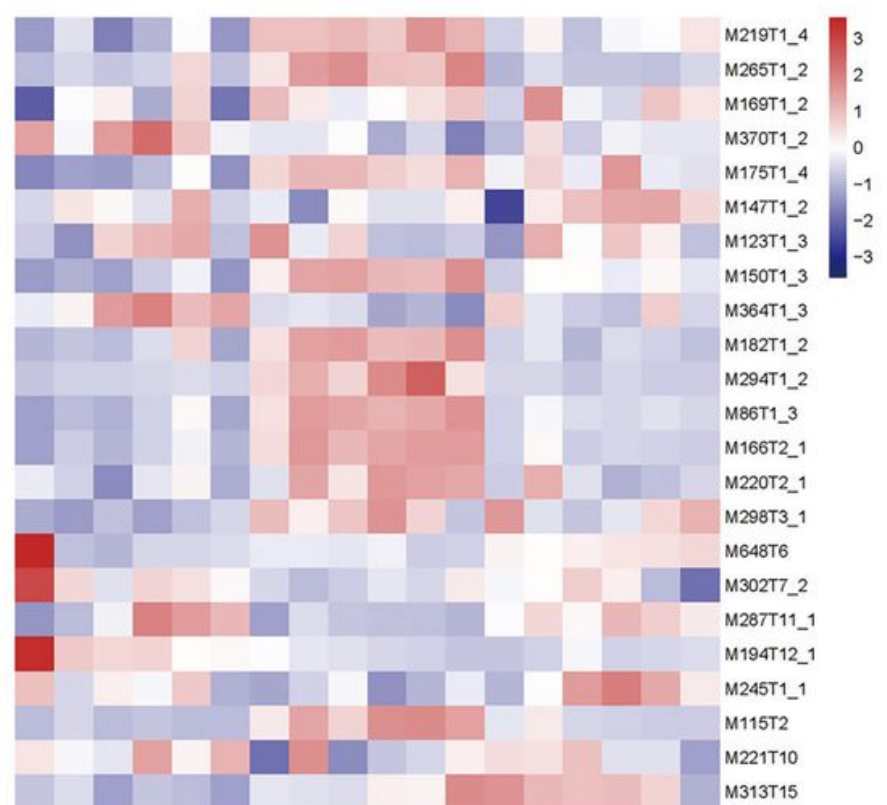

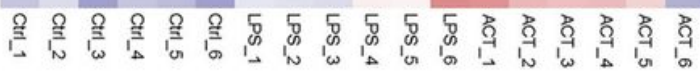

b

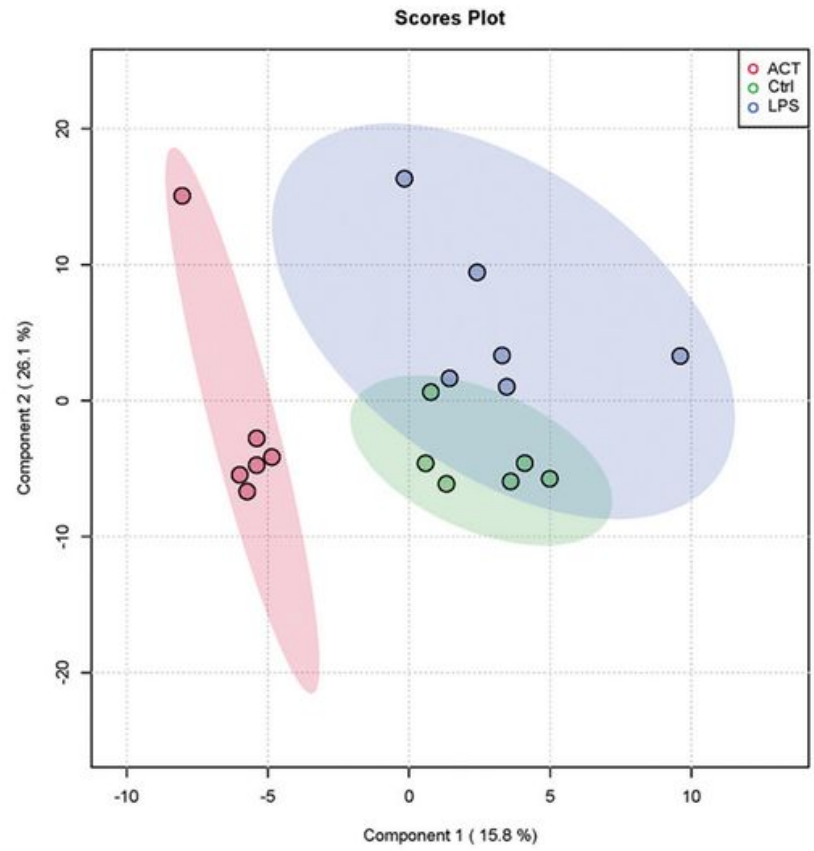

d

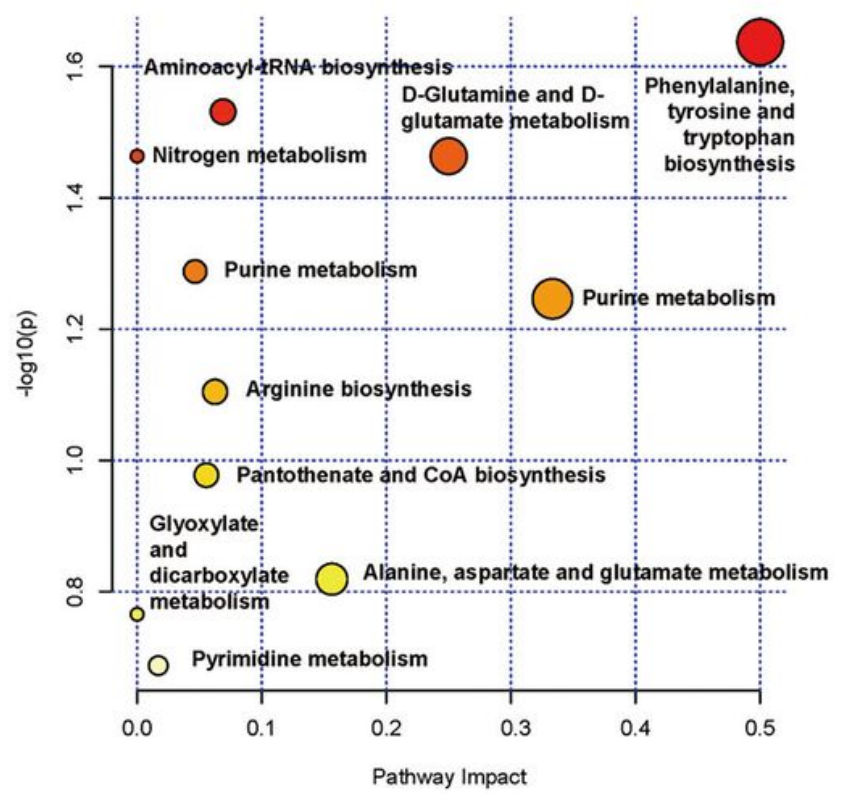

Figure 5

ACT positively regulated the metabolism shifts of BV-2 cells. (a) Principal components analysis (PCA) score plot and (b) partial least squares-discrimination analysis (PLS-DA) score plot for discriminating the 
cell metabolome from Ctrl, LPS and ACT (50 $\mu \mathrm{M})$ groups in positive ion mode. (c) Heat map of the differential metabolites that were altered in LPS group compared to Ctrl group and ACT group compared to LPS group $(n=6)$. (d) The disturbed metabolic pathways in ACT group.

a
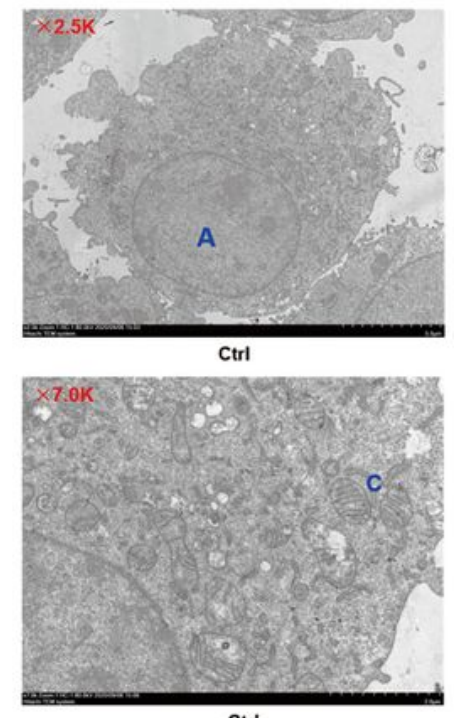

Ctri

b

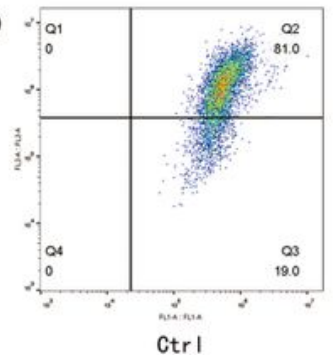

c
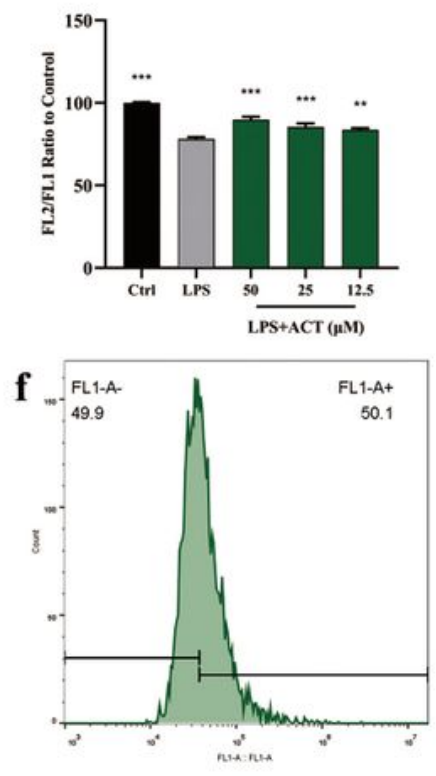

Ctr I
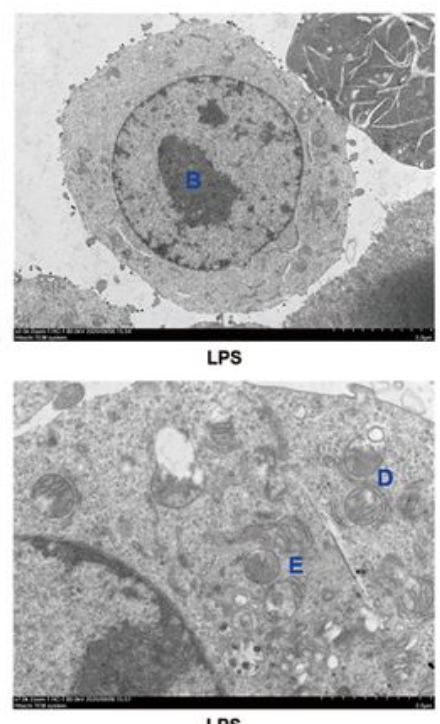

LPS

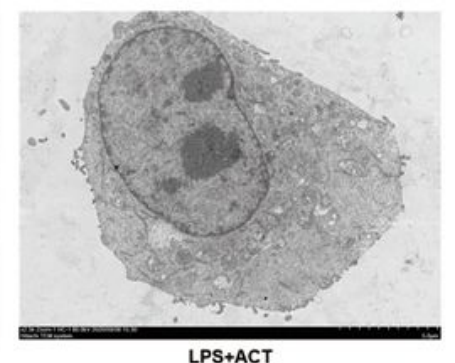

LPS+ACT

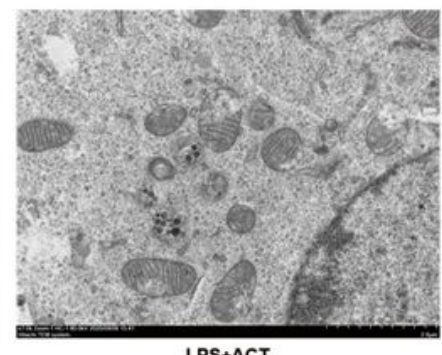

LPS+ACT

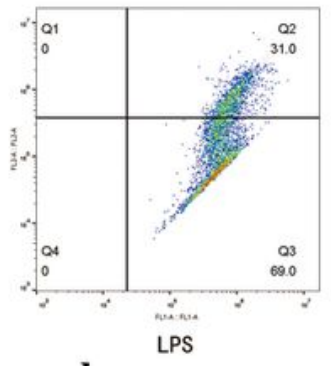

d

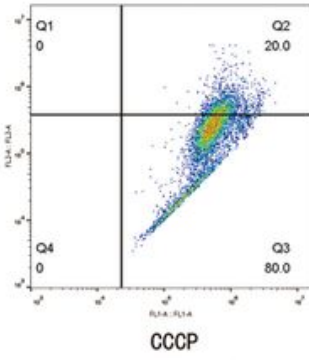

e
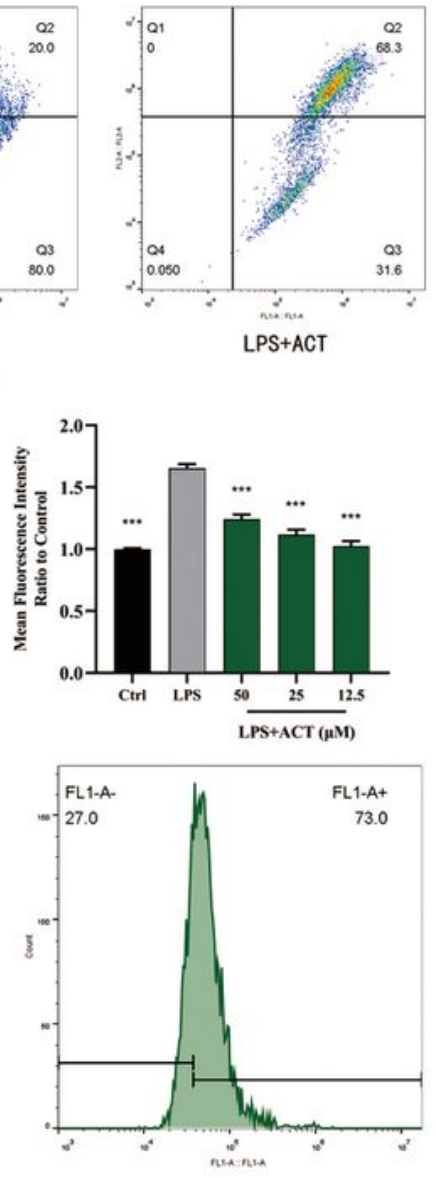

LPS+ACT

Figure 6

Effects of ACT on mitochondrial function in LPS-treated BV-2 cells. (a) Ultrastructural changes in the different groups of BV-2 cells under the transmission electron microscopy. A, normal nucleus. B, nucleus 
chromatin condensation. C, normal mitochondria. D, mitochondrial cristae disarranged or even disappeared. E, swollen and vacuolated mitochondria. (b) The representative images of JC-1 staining. (c) Bar graph of JC-1 red and green fluorescence ratio. (d) Bar graph showing quantified statistics of ATP level. (e) Bar graph of intracellular ROS production. (f) The representative images of DCFH-DA staining. *P囚0.05, **P囚0.01, ***P®0.005, versus the LPS group. $n=3$.

a
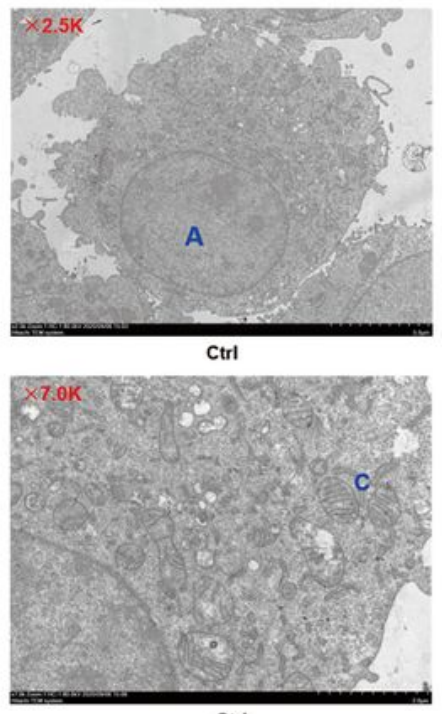

CtrI

b

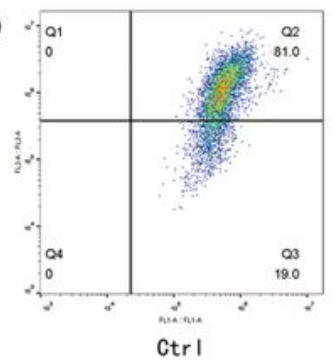

c
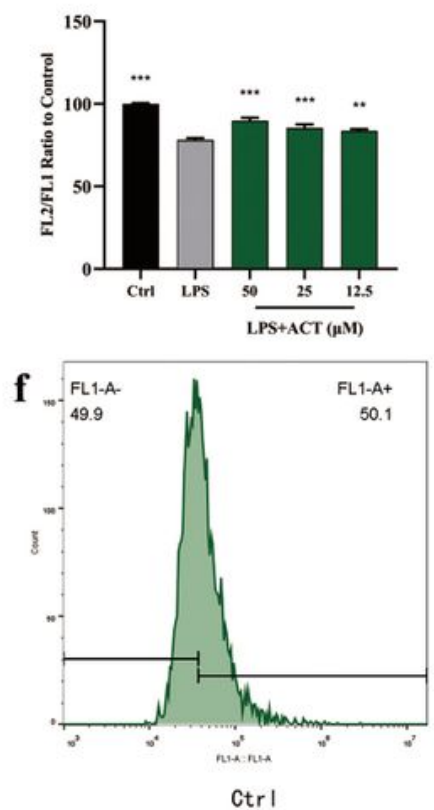
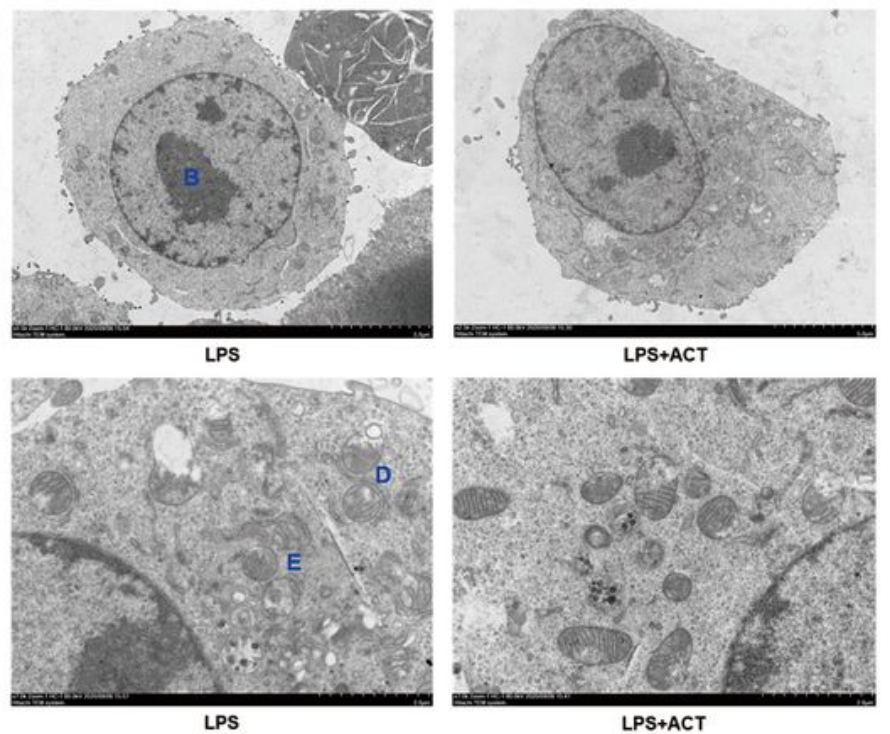

LPS+ACT

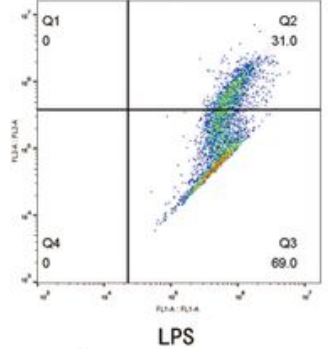

d

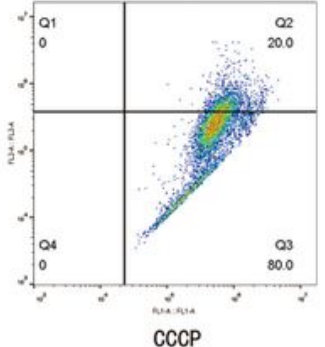

e
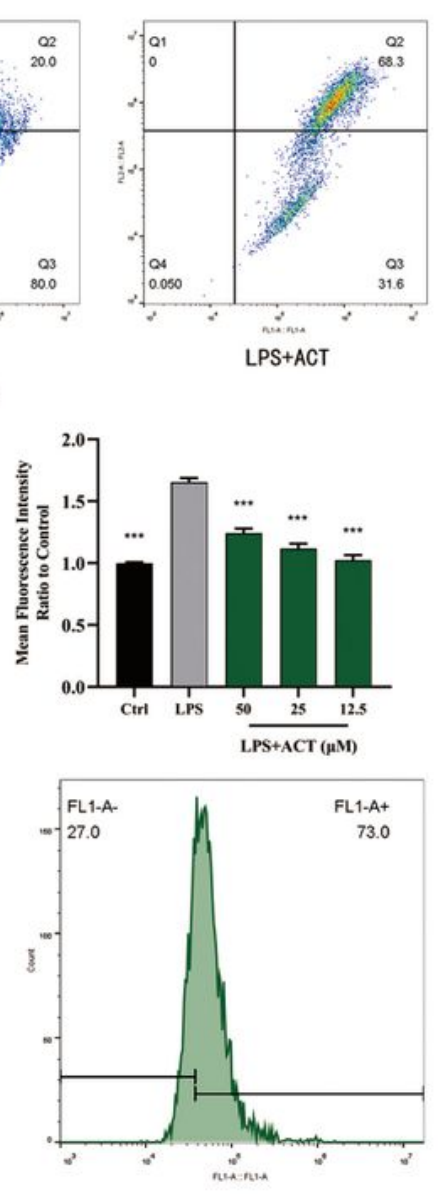

LPS+ACT

Figure 6 
Effects of ACT on mitochondrial function in LPS-treated BV-2 cells. (a) Ultrastructural changes in the different groups of BV-2 cells under the transmission electron microscopy. A, normal nucleus. B, nucleus chromatin condensation. C, normal mitochondria. D, mitochondrial cristae disarranged or even disappeared. E, swollen and vacuolated mitochondria. (b) The representative images of JC-1 staining. (c) Bar graph of JC-1 red and green fluorescence ratio. (d) Bar graph showing quantified statistics of ATP level. (e) Bar graph of intracellular ROS production. (f) The representative images of DCFH-DA staining. $* P \otimes 0.05, * * P \otimes 0.01, * * * P \otimes 0.005$, versus the LPS group. $n=3$.

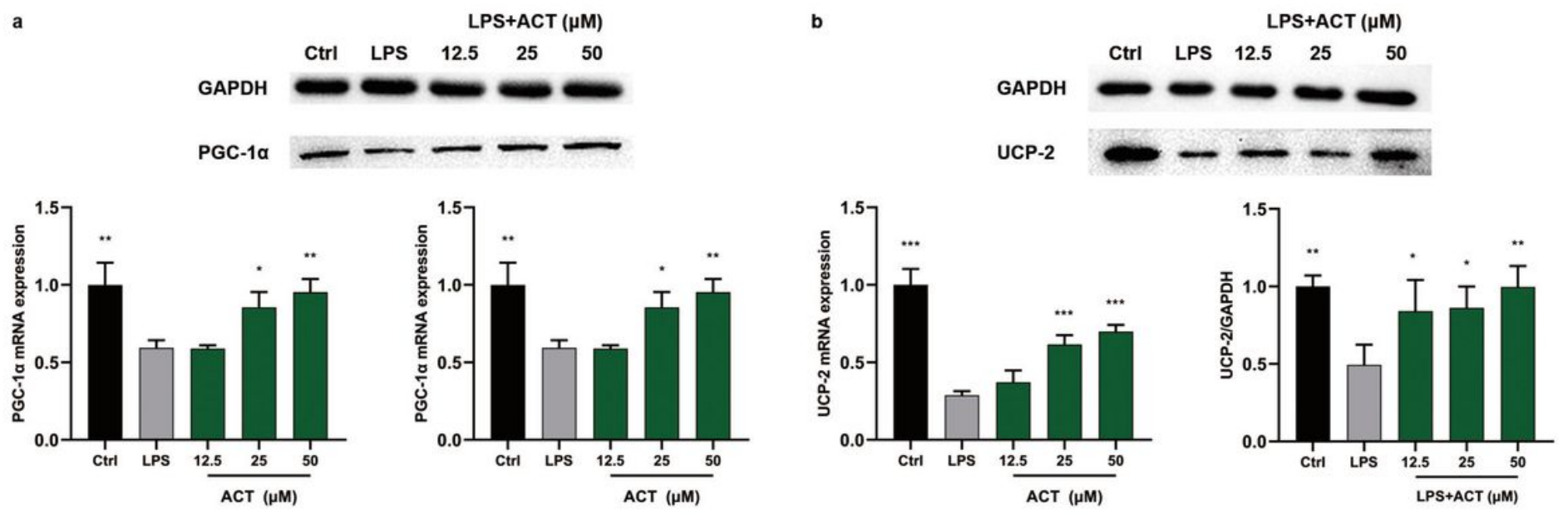

Figure 7

Effect of ACT on PGC-1 a and UCP-2 in LPS-induced BV-2 cells. (a) ACT distinctly decreases the protein and mRNA levels of PGC-1a. (b) ACT distinctly decreases the protein and mRNA levels of UCP-2. *P囚0.05, **P $\otimes 0.01, * * * P \otimes 0.005$, versus the LPS group. $\mathrm{n}=3$.

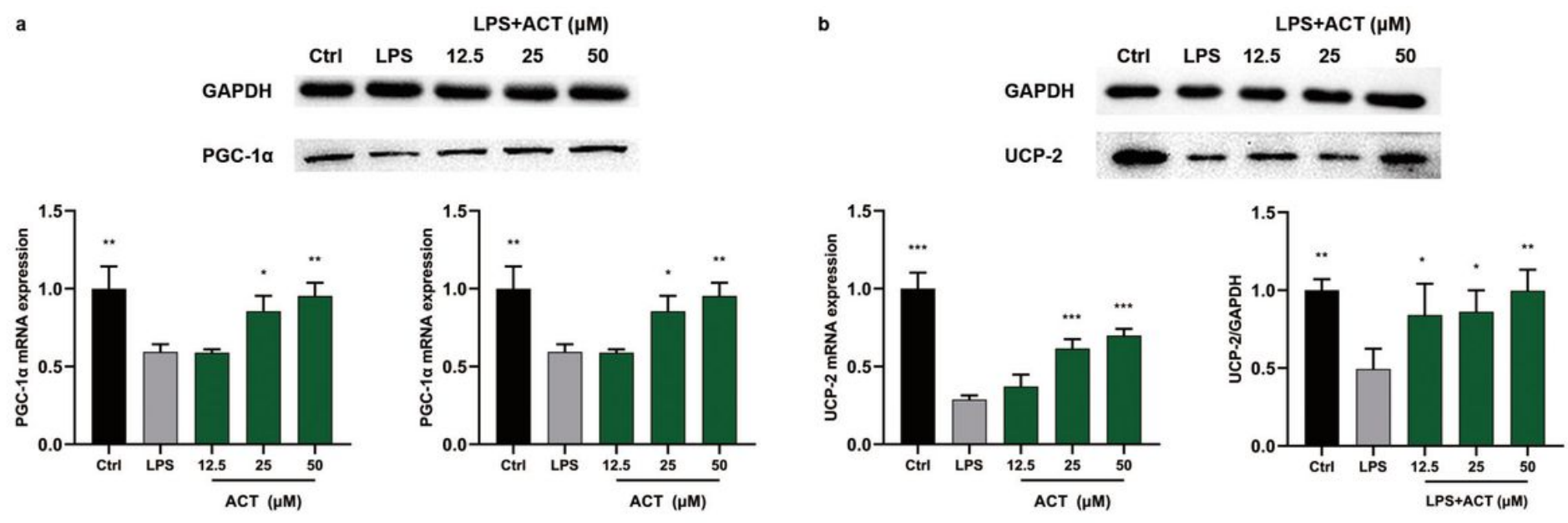

Figure 7

Effect of ACT on PGC-1 $a$ and UCP-2 in LPS-induced BV-2 cells. (a) ACT distinctly decreases the protein and mRNA levels of PGC-1a. (b) ACT distinctly decreases the protein and mRNA levels of UCP-2. *P囚0.05, $\star * P \otimes 0.01, * \star \star P \otimes 0.005$, versus the LPS group. $n=3$. 
a

t-AMPK

p-AMPK

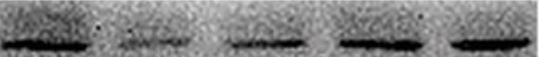

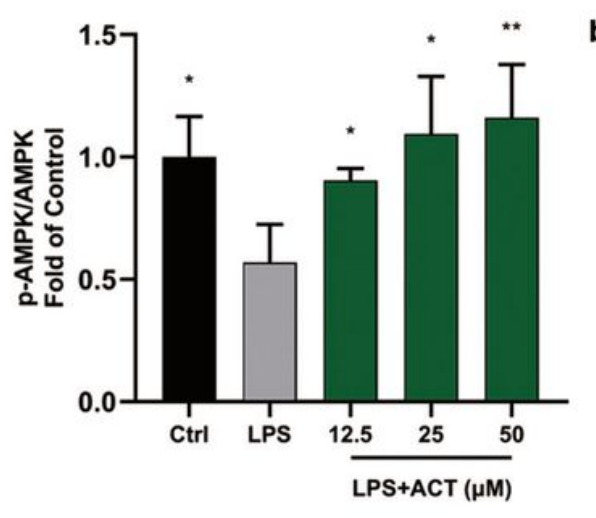

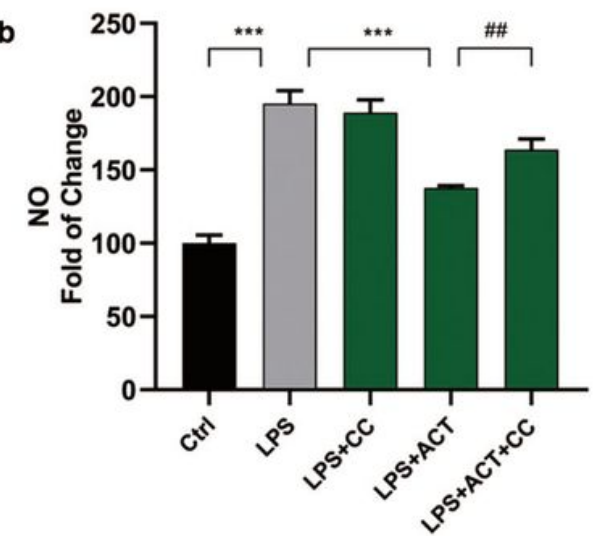

Figure 8

Effect of ACT on AMPK signalling pathways in LPS-induced BV-2 cells. (a) ACT distinctly increases the phosphorylation level of AMPKa. (b) The effect of compound C ( $1 \mu \mathrm{M})$ on NO level. ***P凶0.001, versus the LPS group. \#\#P®0.01, versus the LPS+ACT group. $n=3$.

a

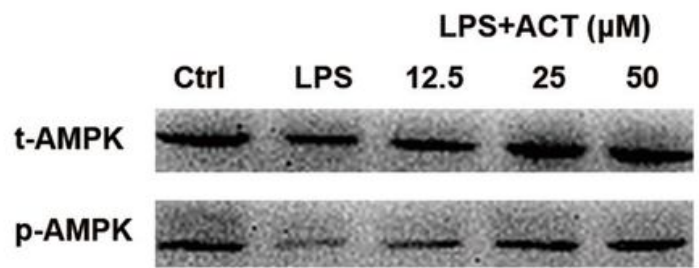

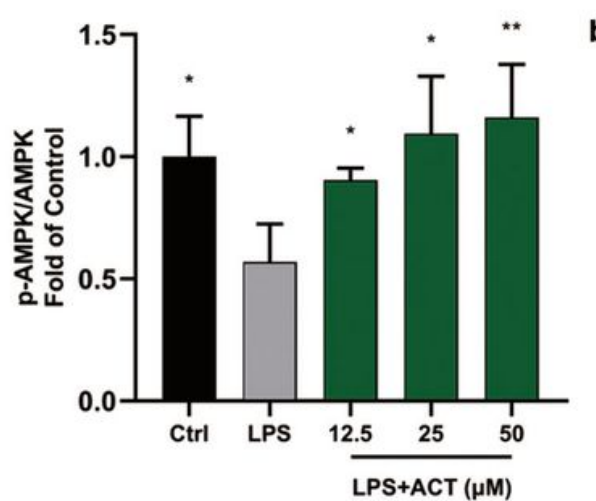

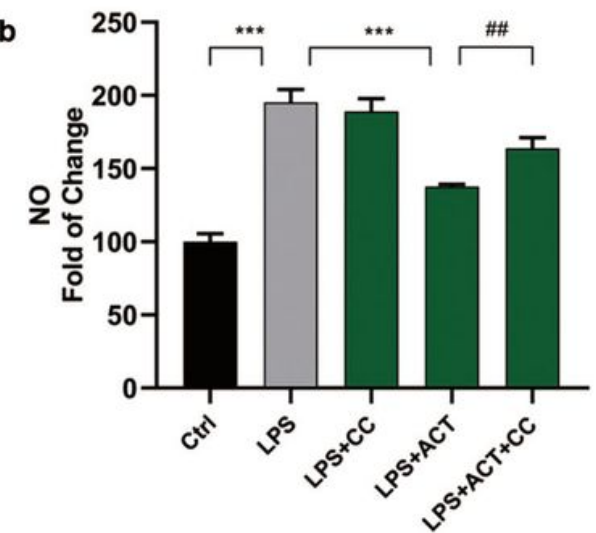

Figure 8

Effect of ACT on AMPK signalling pathways in LPS-induced BV-2 cells. (a) ACT distinctly increases the phosphorylation level of AMPKa. (b) The effect of compound C ( $1 \mu \mathrm{M})$ on NO level. ${ }^{*} \mathrm{P} \otimes 0.001$, versus the LPS group. \#\#P®0.01, versus the LPS+ACT group. $n=3$. 
$\mathbf{a}$

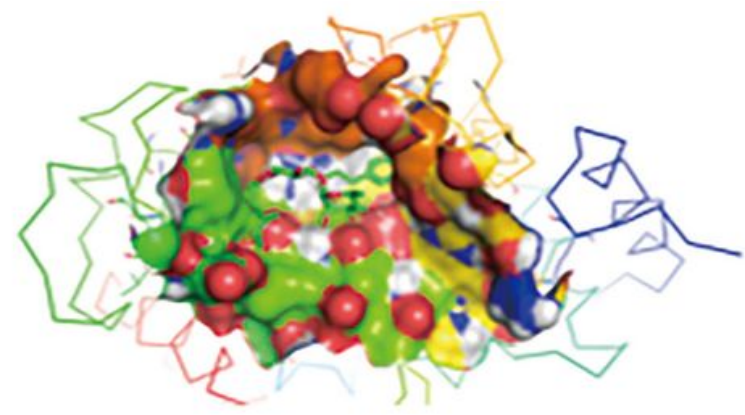

b

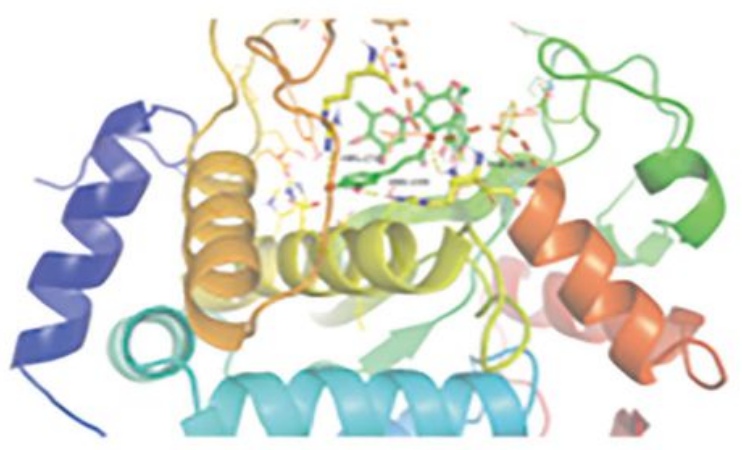

c

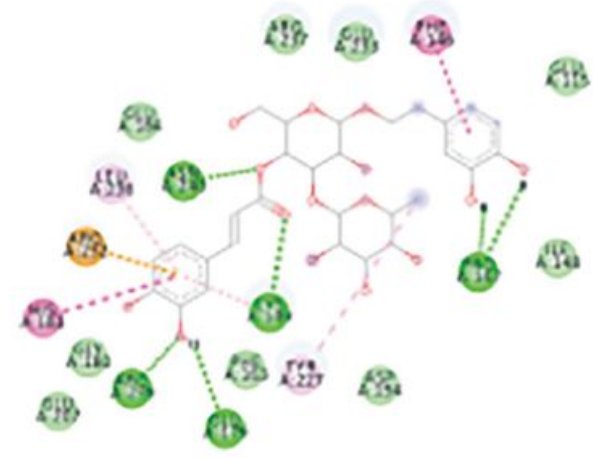

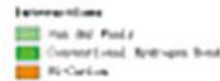

를

NF-kB Affinity: $-8.4 \mathrm{kcal} / \mathrm{mol}$ d

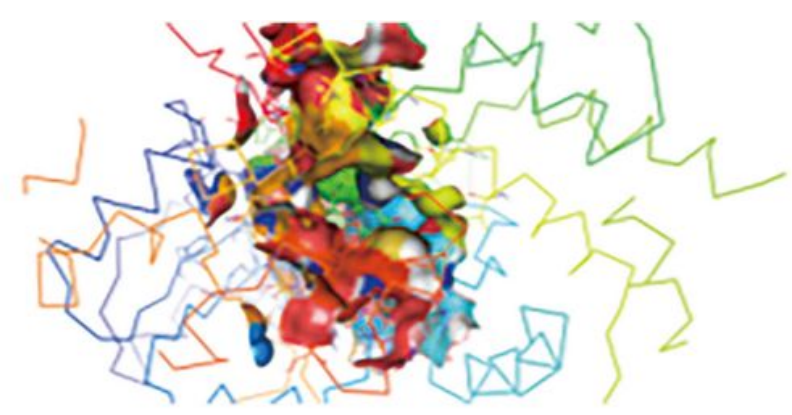

e
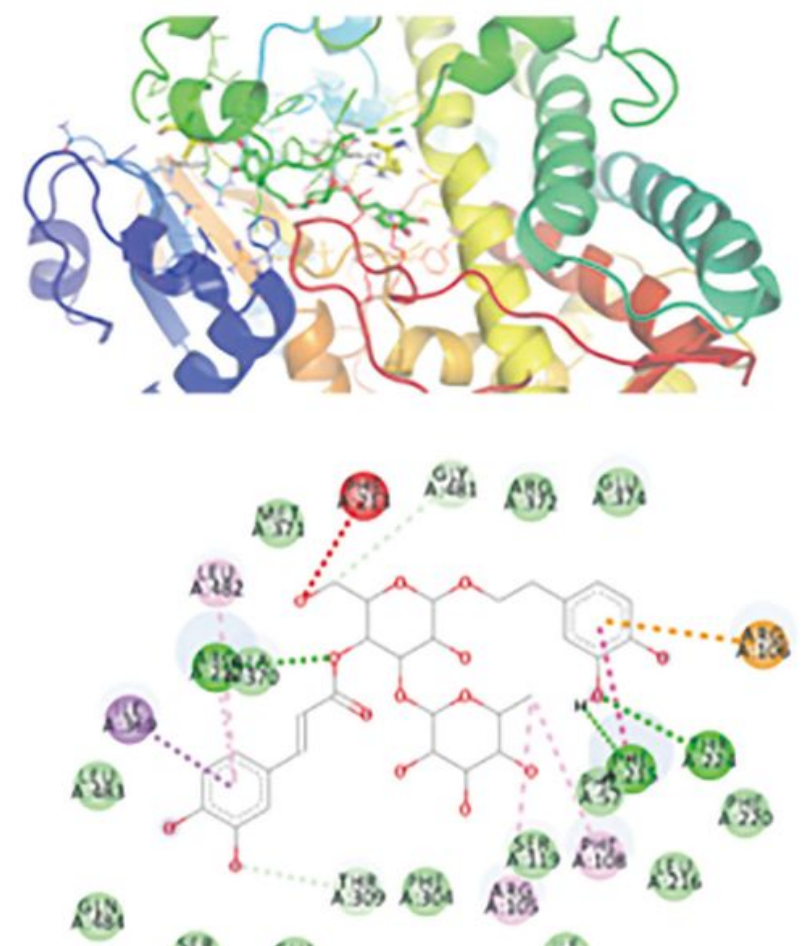

891 280

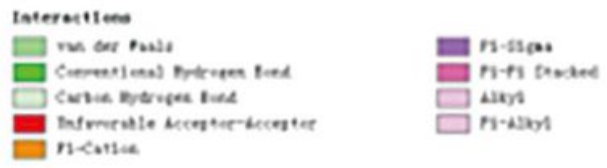

AMPKa Affinity: $-10.8 \mathrm{kcal} / \mathrm{mol}$

Figure 9

ACT bond to and inhibited NF-KB as well as activated AMPKa. (a) A three-dimensional visual of the binding sites between ACT and NF-KB. (b) A two-dimensional close-up of the molecule binding pocket from the side. (c) Amino acid residues. (d) A three-dimensional visual of the binding sites between ACT and AMPKa. (e) A two-dimensional close-up of the molecule binding pocket from the side. (f) Amino acid residues. 
$\mathbf{a}$

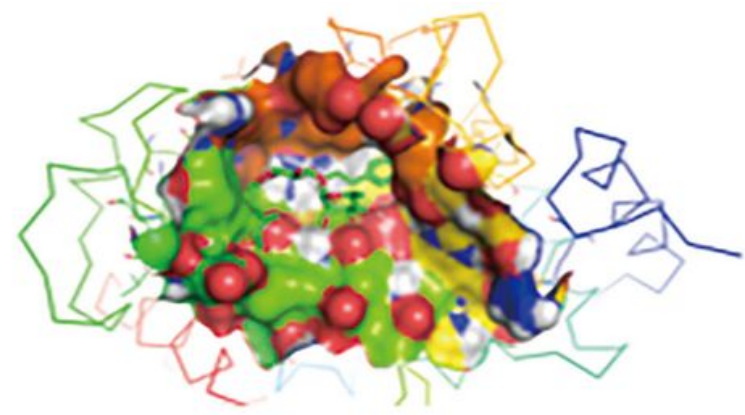

b

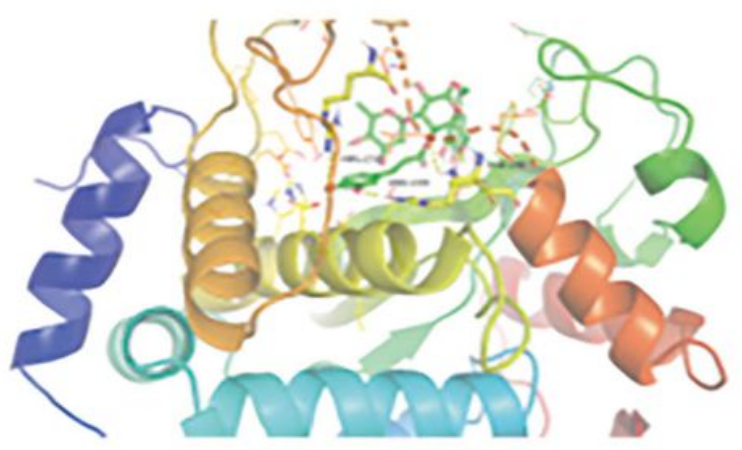

c

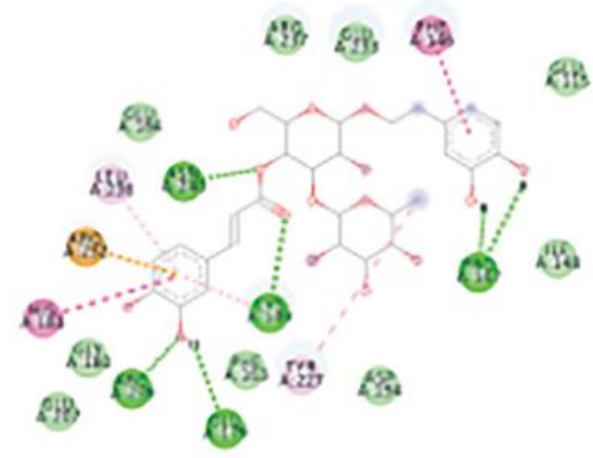

밀

믈

NF-kB Affinity: $-8.4 \mathrm{kcal} / \mathrm{mol}$ d

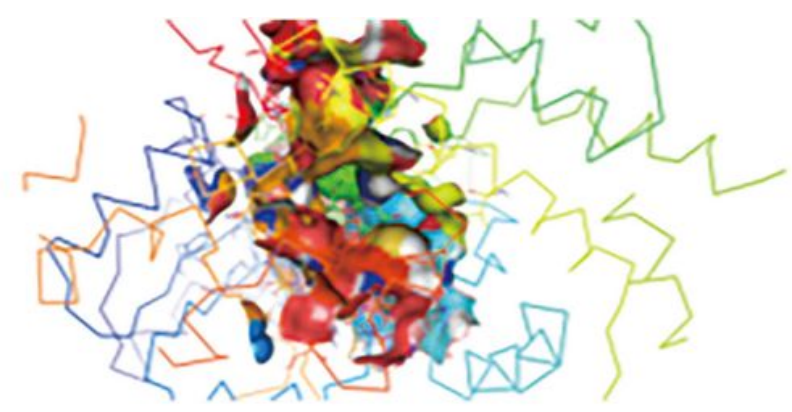

e
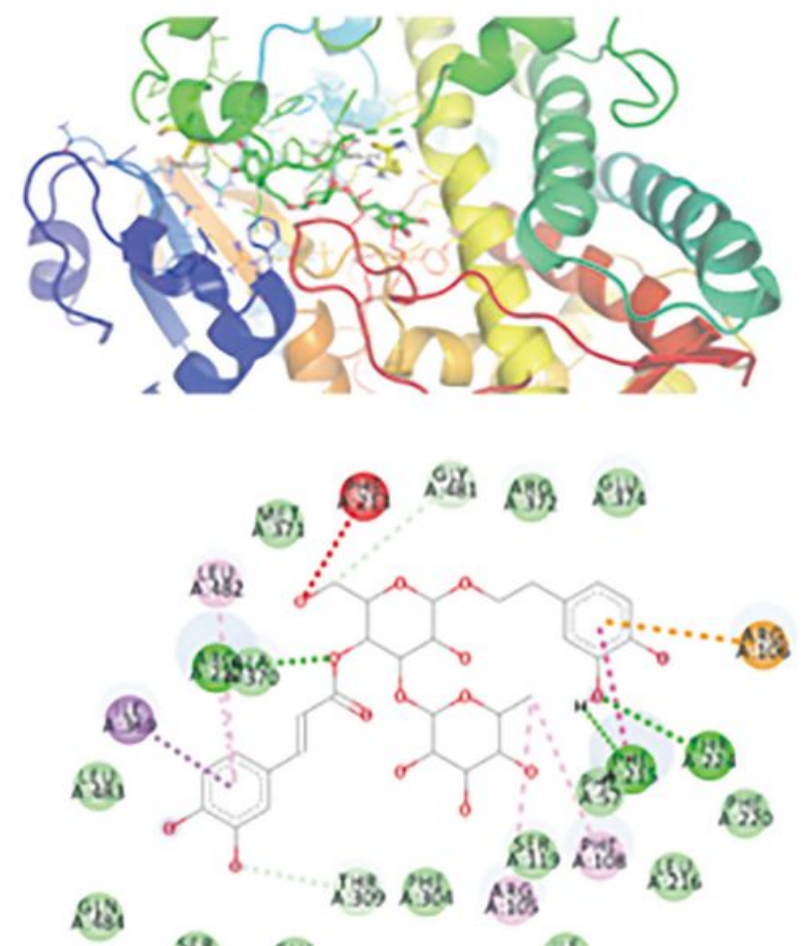

89. 280

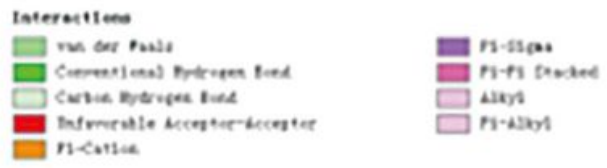

AMPKa Affinity: $-10.8 \mathrm{kcal} / \mathrm{mol}$

Figure 9

ACT bond to and inhibited NF-KB as well as activated AMPKa. (a) A three-dimensional visual of the binding sites between ACT and NF-KB. (b) A two-dimensional close-up of the molecule binding pocket from the side. (c) Amino acid residues. (d) A three-dimensional visual of the binding sites between ACT and AMPKa. (e) A two-dimensional close-up of the molecule binding pocket from the side. (f) Amino acid residues. 


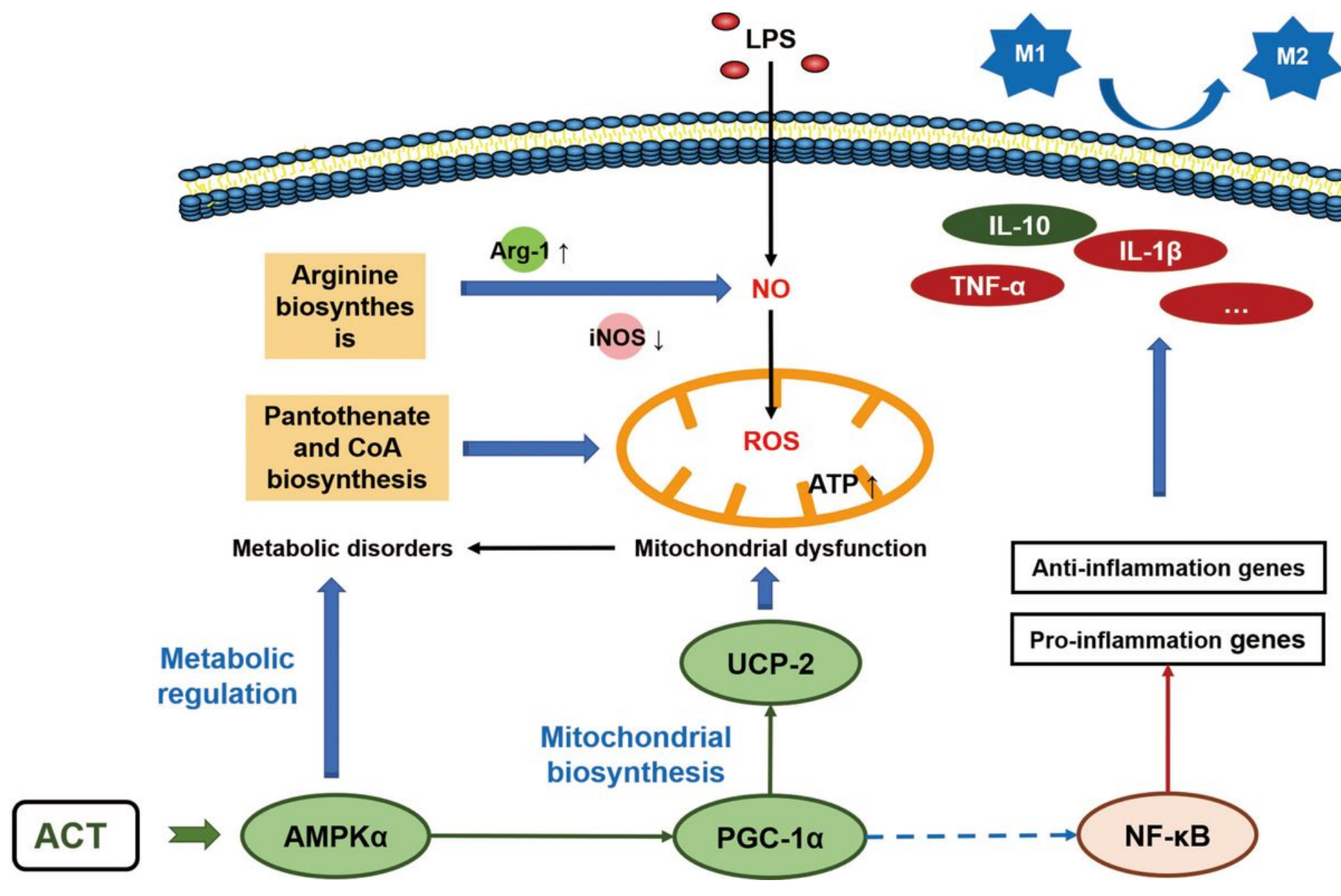

Figure 10

Schematic model of the mechanism by which ACT suppresses LPS-induced M1 polarization via regulating AMPK and NF-KB signalling pathways. 


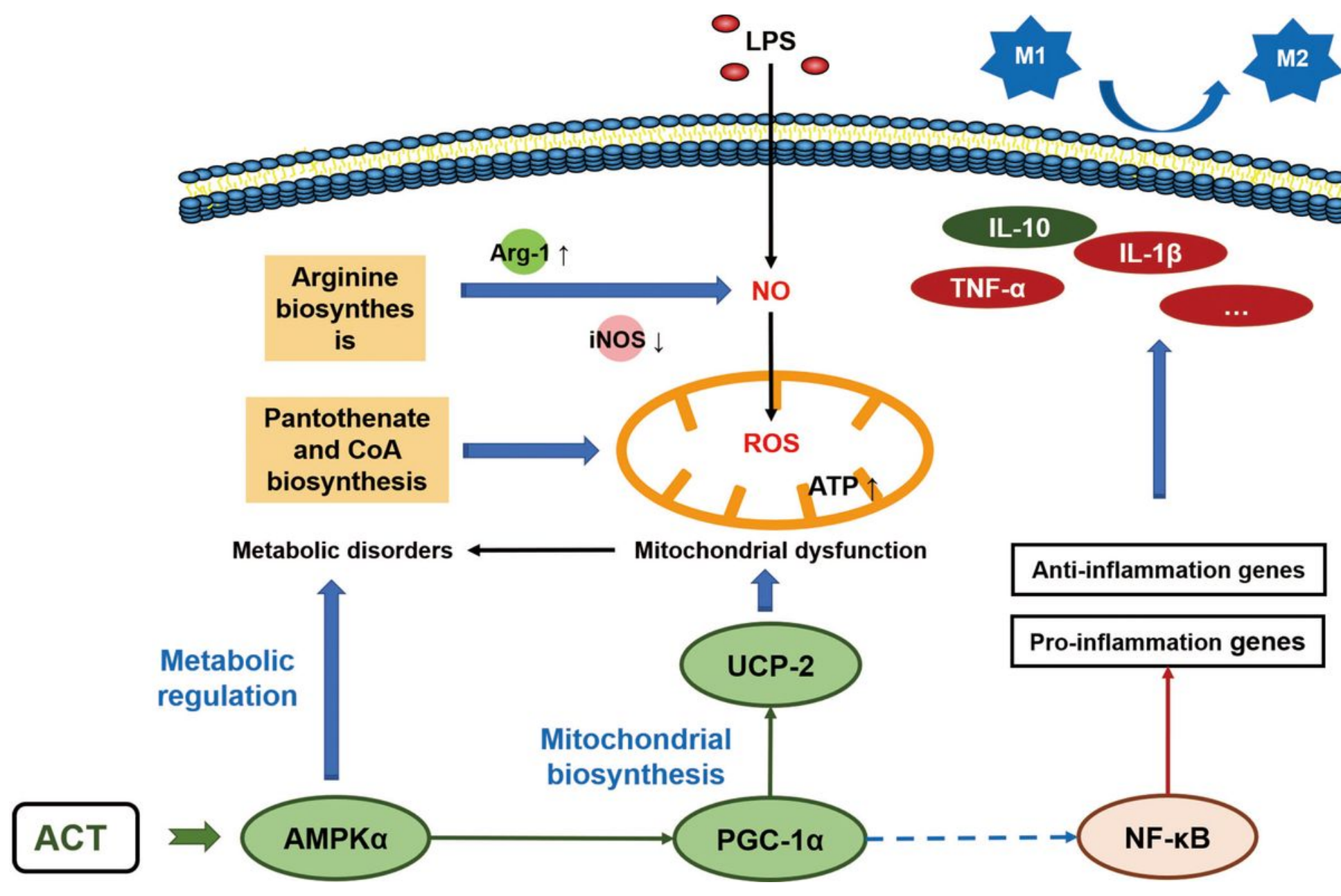

Figure 10

Schematic model of the mechanism by which ACT suppresses LPS-induced M1 polarization via regulating AMPK and NF-KB signalling pathways.

\section{Supplementary Files}

This is a list of supplementary files associated with this preprint. Click to download.

- AMPK.tif

- AMPK.tif

- AdditionalfilesLYQ.doc

- AdditionalfilesLYQ.doc

- GA.tif

- GA.tif

- NFKB.tif

- NFKB.tif

- PGC1A.tif 
- PGC1A.tif

- PNFKB.tif

- PNFKB.tif

- Table.S1.xIsx

- Table.S1.xIsx

- Table.S2.xIsx

- Table.S2.xIsx

- Table.S3.xlsx

- Table.S3.xlsx

- UCP2.tif

- UCP2.tif

- pAMPK.tif

- pAMPK.tif 